RUNHETC-2002-34 MIFP-02-01 Imperial/TP/01-02/27 UPR-1013-T

hep-th/0209193

\title{
Linearly-realised Worldsheet Supersymmetry in pp-wave Backgrounds
}

\author{
M. Cvetič ${ }^{\dagger 1}$, H. Lü ${ }^{\ddagger 2}$, C.N. Pope ${ }^{\ddagger 2}$ and K.S. Stelle ${ }^{\star 3}$ \\ ${ }^{\dagger}$ Department of Physics and Astronomy, \\ Rutgers University, Piscataway, NJ 08855, USA

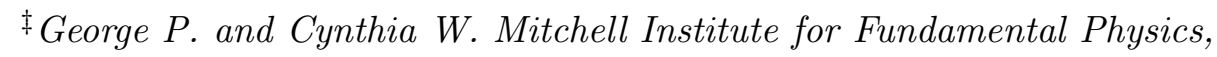 \\ Texas A $6 M$ University, College Station, TX 77843-4242, USA \\ * The Blackett Laboratory, Imperial College, Prince Consort Road, London SW7 2BZ.
}

$\underline{\text { ABSTRACT }}$

We study the linearly-realised worldsheet supersymmetries in the "massive" type II lightcone actions for pp-wave backgrounds. The pp-waves have have $16+N_{\text {sup }}$ Killing spinors, comprising 16 "standard" Killing spinors that occur in any wave background, plus $N_{\text {sup }}$ "supernumerary" Killing spinors $\left(0 \leq N_{\text {sup }} \leq 16\right)$ that occur only for special backgrounds. We show that only the supernumerary Killing spinors give rise to linearly-realised worldsheet supersymmetries after light-cone gauge fixing, while the 16 standard Killing spinors describe only non-linearly realised inhomogeneous symmetries. We also study the type II actions in the physical gauge, and we show that although in this case the actions are not free, there are now linearly-realised supersymmetries coming both from the standard and the supernumerary Killing spinors. In the physical gauge, there are no mass terms for any worldsheet degrees of freedom, so the masses appearing in the light-cone gauge may be viewed as gauge artefacts. We obtain type IIA and IIB supergravity solutions describing solitonic strings in pp-wave backgrounds, and show how these are related to the physicalgauge fundamental string actions. We study the supersymmetries of these solutions, and find examples with various numbers of Killing spinors, including total numbers that are odd.

\footnotetext{
${ }^{1}$ On sabbatical leave from the University of Pennsylvania. Research supported in part by DOE grant DOE-FG02-95ER40893, NATO linkage grant No. 97061 and Class of 1965 Endowed Term Chair.

2 Research supported in part by DOE grant DE-FG03-95ER40917

${ }^{3}$ Research supported in part by the EC under RTN contract HPRN-CT-2000-00131.
} 


\section{Contents}

1 Introduction $\quad 2$

2 Type IIA Green-Schwarz Action 4

2.1 Kappa symmetry and supersymmetry in type IIA . . . . . . . . . . . . . . 4

2.2 Type IIA worldsheet supersymmetry in the light-cone gauge . . . . . . . . . 6

3 Type IIB Green-Schwarz Action 9

3.1 Kappa symmetry and supersymmetry in type IIB . . . . . . . . . . . . . . . 9

3.2 Type IIB worldsheet supersymmetry in the light-cone gauge . . . . . . . . . 11

4 Worldsheet Supersymmetry in pp-wave Backgrounds 12

4.1 Killing spinors in pp-wave backgrounds . . . . . . . . . . . . . . . . . . 12

4.2 Linearly-realised worldsheet supersymmetries . . . . . . . . . . . . . . . . 14

5 Green-Schwarz Actions for pp-waves in Physical Gauge 15

5.1 Type IIA worldsheet supersymmetries in the physical gauge . . . . . . . . 15

5.2 Type IIB worldsheet supersymmetries in the physical gauge . . . . . . . . 18

5.3 Absence of mass-terms in the physical gauge . . . . . . . . . . . . . . 21

6 Strings in pp-wave Backgrounds: Supergravity Solutions 22

6.1 The type IIB supergravity solution . . . . . . . . . . . . . . . . . 23

6.2 Supersymmetry of the type IIB string on a pp-wave . . . . . . . . . . . 24

6.3 The type IIA supergravity solution . . . . . . . . . . . . . . . . 26

6.4 Supersymmetry of the type IIA string on a pp-wave $\ldots \ldots \ldots$

6.5 Strings on pp-waves with odd numbers of Killing spinors . . . . . . . . . . 28

6.6 Further solutions for strings in pp-waves . . . . . . . . . . . . . . 30

7 Conclusions $\quad 31$ 


\section{Introduction}

The pp-wave configuration $[1,2,3]$ that arises as a Penrose limit [4] of the $\operatorname{AdS}_{5} \times S^{5}$ solution in string theory gives rise to an exactly-solvable free massive worldsheet-supersymmetric string action in the light-cone gauge $[5,6]$, which can provide insights into aspects of the AdS/CFT correspondence [6]. More general pp-wave solutions of a similar kind also exist [7, $8,9,10]$, which may or may not be obtainable from any Penrose limit. They again give rise to exactly-solvable free massive string actions. The Penrose limit of $\operatorname{AdS}_{5} \times S^{5}$ is maximally supersymmetric, with 32 Killing spinors. Typically, the other pp-wave solutions have less supersymmetry, although every pp-wave admits at least 16 Killing spinors, regardless of the specific details of its construction. In fact the Killing spinors divide into two categories, which in the terminology of $[7,8]$ are the 16 "standard" Killing spinors that every ppwave has, and the possible further "supernumerary" Killing spinors, whose number $N_{\text {sup }}$ $\left(0 \leq N_{\text {sup }} \leq 16\right)$ depends upon the details of the solution.

An important question arises concerning the supersymmetry of the light-cone string action. Prior to gauge fixing, the Green-Schwarz action has a local kappa symmetry and a rigid spacetime supersymmetry, with a number of parameters equal to the number of Killing spinors in the target spacetime. After imposing the light-cone gauge conditions, the kappa symmetry and spacetime supersymmetry transmute into rigid worldsheet supersymmetries of the string action. Of particular interest are the worldsheet supersymmetries that are linearly realised, since they establish a pairing between the bosonic and fermionic degrees of freedom, and they can imply relations between the masses of the bosons and the fermions.

It was observed in $[7,8]$ that in general, for a pp-wave that has only the 16 standard Killing spinors, the mass terms for the bosons and the fermions are unequal, and thus evidently there could be no linearly-realised worldsheet supersymmetries. ${ }^{1}$ By contrast, it was observed that if there are supernumerary Killing spinors that in addition are independent of the $X^{+}$coordinate (and hence are independent of the worldsheet time coordinate in lightcone gauge, and so commute with the Hamiltonian) then the boson and fermion masses are related. This led to the conjecture in $[7,8]$ that it is only the supernumerary Killing spinors that can give rise to linearly-realised worldsheet supersymmetries in the string action in the light-cone gauge.

One of the main purposes of the present paper is to prove this conjecture, by showing that when one constructs the light-cone-gauge string action in a pp-wave background,

\footnotetext{
${ }^{1}$ Or at least none that commute with the Hamiltonian.
} 
linearly-realised worldsheet supersymmetries are indeed associated purely with the supernumerary Killing spinors that arise in special pp-wave backgrounds. By contrast, we show that the 16 standard Killing spinors do not give rise to any linearly-realised worldsheet supersymmetries. We prove these results by showing that the projection conditions for linearly-realised worldsheet supersymmetries that arise from fixing the kappa symmetry of the Green-Schwarz string action in the light-cone gauge eliminate all the standard Killing spinors in the pp-wave, but allow all the supernumerary Killing spinors to remain. This shows that it is precisely the supernumerary Killing spinors that are associated with linearlyrealised worldsheet supersymmetries in the light-cone gauge.

A different approach to the question of linearly-realised worldsheet supersymmetry is to construct a classical supergravity solution describing a solitonic string on a pp-wave background. The dynamics of the string on this background arise from considering the Goldstone modes associated with the breaking of symmetries of the pp-wave background when the string is present. We construct such intersecting pp-wave/string solutions. A puzzle is that these always preserve some non-vanishing fraction of the supersymmetry, even in cases where the pp-wave by itself would have no supernumerary supersymmetries. One expects to find, therefore, that the Goldstone boson and fermion modes are still related by a linearly-realised supersymmetry, associated with the residual unbroken supersymmetry of the pp-wave/string solution, even in cases without supernumerary supersymmetries. This would then imply that the associated string action, which describes the dynamics of the Goldstone modes, should have linearly realised worldsheet supersymmetry regardless of whether or not there exist supernumerary Killing spinors in the pure pp-wave background.

The resolution of this apparent discrepancy with the previous light-cone discussion is that the solution with a string on a pp-wave leads to Goldstone modes describing the string dynamics in the physical gauge and not the light-cone gauge. (In the physical gauge, two of the ten target spacetime coordinates are set equal to the two string worldsheet coordinates $(\tau, \sigma)$, rather than setting $X^{+}=\tau$ as one does in light-cone gauge.) We are therefore led to re-examine the string action in the physical gauge, to see what conclusions one then reaches about linearly-realised worldsheet supersymmetries in a pp-wave background.

We study this issue by starting from the Green-Schwarz string action, and fixing the kappa symmetry in physical gauge, in order to derive the residual linearly-realised worldsheet supersymmetry. We find that, unlike the analogous calculation in light-cone gauge, where the projection conditions remove all 16 of the standard Killing spinors of a generic ppwave background, in physical gauge the projection can instead preserve a certain fraction of 
the standard Killing spinors. It also eliminates some of the supernumerary Killing spinors, while preserving others. Thus in the physical gauge, there is linearly-realised worldsheet supersymmetry for any pp-wave background, with enhanced worldsheet supersymmetry if there are also supernumerary Killing spinors.

In section 2 we consider the type IIA Green-Schwarz action, up to terms of quadratic order in fermions, and we exhibit the kappa symmetry and spacetime supersymmetry transformations. We then derive the rigid worldsheet supersymmetries that survive after imposing the light-cone gauge conditions. In section 3, we give an analogous discussion for the type IIB Green-Schwarz action. In section 4, we show how the projection conditions for linearly-realised worldsheet supersymmetries in the type IIA and type IIB light-cone actions are compatible with those satisfied by the supernumerary Killing spinors, but they are orthogonal to the projection conditions satisfied by the 16 standard Killing spinors. In section 5 we turn to an analysis of the physical gauge, deriving the residual worldsheet supersymmetries after gauge-fixing. The projection conditions on the residual supersymmetries turn out to be compatible with standard as well as supernumerary Killing spinors in the physical gauge. In section 6, we construct new supergravity solutions describing strings in pp-wave backgrounds, and show how these provide realisations for string actions in pp-wave backgrounds in the physical gauge. Various unusual fractions of supersymmetry can be achieved in the string/pp-wave solutions, including odd numbers of Killing spinors.

\section{Type IIA Green-Schwarz Action}

\subsection{Kappa symmetry and supersymmetry in type IIA}

The explicit component form of the Green-Schwarz action for the type IIA string on an arbitrary bosonic background, up to and including terms quartic in the fermionic coordinates, was derived in [11] This result was obtained by double dimensional reduction of an explicit component form of the supermembrane action in $D=11$ that was obtained in [12]. The reduction to the type IIA string that was performed in [11] was a component analogue of the superfield reduction performed in [13]. The type IIA action found in [11] is

$$
\begin{aligned}
\mathcal{L}_{2}= & -\frac{1}{2} \sqrt{-h} h^{i j} \partial_{i} X^{\mu} \partial_{j} X^{\nu} g_{\mu \nu}+\frac{1}{2} \epsilon^{i j} \partial_{i} X^{\mu} \partial_{j} X^{\nu} A_{\mu \nu} \\
& -\mathrm{i} \bar{\theta} \beta^{i j} \Gamma_{\mu} D_{j} \theta \partial_{i} X^{\mu}+\frac{\mathrm{i}}{8} \partial_{i} X^{\mu} \partial_{j} X^{\nu} \bar{\theta} \beta^{i j} \Gamma_{11} \Gamma_{\mu}^{\rho \sigma} \theta F_{\nu \rho \sigma} \\
& -\frac{\mathrm{i}}{16} \partial_{i} X^{\mu} \partial_{j} X^{\nu} e^{\phi} \bar{\theta} \beta^{i j}\left(\Gamma_{11} \Gamma_{\mu} \Gamma^{\rho \sigma} \Gamma_{\nu} F_{\rho \sigma}+\frac{1}{12} \Gamma_{\mu} \Gamma^{\rho \sigma \lambda \tau} \Gamma_{\nu} F_{\rho \sigma \lambda \tau}\right) \theta,
\end{aligned}
$$


where

$$
\beta^{i j} \equiv \sqrt{-h} h^{i j}-\epsilon^{i j} \Gamma_{11}, \quad D_{i} \theta \equiv \partial_{i} \theta+\frac{1}{4} \partial_{i} X^{\mu} \omega_{\mu}^{m n} \Gamma_{m n} \theta
$$

The field strengths are given by

$$
F_{(4)}=d A_{(3)}-A_{(1)} \wedge d A_{(2)}, \quad F_{(3)}=d A_{(2)}, \quad F_{(2)}=d A_{(1)}
$$

We can show that this action is invariant, up to the quadratic order in $\theta$ to which we are working, under local kappa-symmetry transformations given by

$$
\delta \theta=(1+\Gamma) \kappa, \quad \delta X^{\mu}=-\mathrm{i} \bar{\theta} \Gamma^{\mu} \delta \theta
$$

To the order we are working, the matrix $\Gamma$ is given by

$$
\Gamma=\frac{1}{2 \sqrt{-h}} \epsilon^{i j} \partial_{i} X^{\mu} \partial_{j} X^{\nu} \Gamma_{\mu \nu} \Gamma_{11}
$$

We can also show that the action is invariant, up to the relevant order in $\theta$, under rigid supersymmetry transformations, given by

$$
\delta \theta=\epsilon, \quad \delta X^{\mu}=\mathrm{i} \bar{\theta} \Gamma^{\mu} \epsilon,
$$

where $\epsilon$ is a Killing spinor of the type IIA supergravity background. Specifically, we find that under (6), the Lagrangian (1) varies to give, up to total derivatives,

$$
\delta \mathcal{L}=-2 \mathrm{i} \partial_{i} X^{\mu} \partial_{j} X^{\nu} \bar{\theta} \beta^{i j} \Gamma_{\mu} \mathcal{D}_{\nu} \epsilon
$$

where $\mathcal{D}_{\mu}$ is the supercovariant derivative, given by

$$
\mathcal{D}_{\mu}=\nabla_{\mu}+\frac{1}{8} \Gamma_{11} \Gamma^{\rho \sigma} F_{\mu \rho \sigma}-\frac{1}{16} e^{\phi}\left(\Gamma_{11} \Gamma^{\rho \sigma} F_{\rho \sigma}-\frac{1}{12} \Gamma^{\rho \sigma \lambda \tau} F_{\rho \sigma \lambda \tau}\right) \Gamma_{\mu}
$$

where $\nabla_{\mu} \equiv \partial_{\mu}+\frac{1}{4} \omega_{\mu}^{m n} \Gamma_{m n}$. In the string-frame metric that we are using here, the supersymmetry transformation law for the gravitini is given by $\delta \psi_{\mu}=\mathcal{D}_{\mu} \epsilon$, and so the Green-Schwarz action is invariant under (6) if $\epsilon$ is a Killing spinor.

We may also define the derivative

$$
\widetilde{\mathcal{D}}_{i}=D_{i}+\partial_{i} X^{\mu}\left[\frac{1}{8} \Gamma_{11} \Gamma^{\rho \sigma} F_{\mu \rho \sigma}-\frac{1}{16} e^{\phi}\left(\Gamma_{11} \Gamma^{\rho \sigma} F_{\rho \sigma}-\frac{1}{12} \Gamma^{\rho \sigma \lambda \tau} F_{\rho \sigma \lambda \tau}\right) \Gamma_{\mu}\right]
$$

When acting on a spinor function of $X^{\mu}$ such as $\epsilon$, this reduces to $\widetilde{\mathcal{D}}_{i} \epsilon=\partial_{i} X^{\mu} \mathcal{D}_{\mu} \epsilon$. In terms of $\widetilde{\mathcal{D}}_{i}$, we can write the Green-Schwarz Lagrangian (1) as

$$
\mathcal{L}_{2}=-\frac{1}{2} \sqrt{-h} h^{i j} \partial_{i} X^{\mu} \partial_{j} X^{\nu} g_{\mu \nu}+\frac{1}{2} \epsilon^{i j} \partial_{i} X^{\mu} \partial_{j} X^{\nu} A_{\mu \nu}-\mathrm{i} \partial_{i} X^{\mu} \bar{\theta} \beta^{i j} \Gamma_{\mu} \widetilde{\mathcal{D}}_{j} \theta
$$




\subsection{Type IIA worldsheet supersymmetry in the light-cone gauge}

We begin with a review of how one imposes the light-cone gauge condition in the background of a pp-wave. The covariant Green-Schwarz action can be written as

$$
\mathcal{L}=-\frac{1}{2} \sqrt{-h} h^{i j} \Pi_{i}^{m} \Pi_{j}^{n} \eta_{m n}-\frac{1}{2} \epsilon^{i j} \partial_{i} Z^{M} \partial_{j} Z^{N} A_{N M}
$$

where $\Pi_{i}^{m}=\partial_{i} Z^{M} E_{M}^{m}, Z^{M}=\left(X^{\mu}, \theta_{\alpha}\right)$, and the relevant supervielbeins are given, up to quadratic order in fermion coordinates $\theta$, by

$$
\begin{aligned}
E_{\mu}^{m}= & e_{\mu}^{m}+\frac{\mathrm{i}}{4} \omega_{\mu}^{p q} \bar{\theta} \Gamma^{m} \Gamma_{p q} \theta-\frac{\mathrm{i}}{8} \bar{\theta} \Gamma_{11} \Gamma^{m} \Gamma^{p q} \theta F_{\mu p q}+\frac{\mathrm{i}}{16} e^{\phi} \bar{\theta} \Gamma_{11} \Gamma^{m} \Gamma^{p q} \Gamma_{\mu} \theta F_{p q} \\
& +\frac{\mathrm{i}}{192} e^{\phi} \bar{\theta} \Gamma^{m} \Gamma^{p_{1} \cdots p_{4}} \Gamma_{\mu} \theta F_{p_{1} \cdots p_{4}}, \\
E_{\alpha}^{m}= & -\mathrm{i}\left(\bar{\theta} \Gamma^{m}\right)_{\alpha} .
\end{aligned}
$$

The worldsheet metric is given by

$$
h_{i j}=\Pi_{i}^{m} \Pi_{j}^{n} \eta_{m n}
$$

There is a local worldsheet (kappa) symmetry and a rigid spacetime supersymmetry, ${ }^{2}$ with parameters $\kappa$ and $\epsilon$ respectively,

$$
\delta \theta=(1+\Gamma) \kappa+\epsilon, \quad \delta X^{\mu}=-\mathrm{i} \bar{\theta} \Gamma^{\mu}(1+\Gamma) \kappa+\mathrm{i} \bar{\theta} \Gamma^{\mu} \epsilon,
$$

where

$$
\Gamma=\frac{1}{2 \sqrt{-h}} \epsilon^{i j} \Pi_{i}^{m} \Pi_{j}^{n} \Gamma_{m n} \Gamma_{11}
$$

(Note that we can write $\delta X^{\mu}=-\mathrm{i} \bar{\theta} \Gamma^{\mu} \delta \theta+2 \mathrm{i} \bar{\theta} \Gamma^{\mu} \epsilon$.) One can straightforwardly establish from the definitions that $\Gamma^{2}=1$ and $\operatorname{tr} \Gamma=0$, so $(1+\Gamma)$ projects out half the components of $\kappa$.

In the light-cone gauge one imposes

$$
X^{+}=\tau, \quad \Gamma^{+} \theta=0, \quad \sqrt{-h} h^{i j}=\eta^{i j} .
$$

From (12) and the other definitions above, it therefore follows that in cases where the bosonic vielbein satisfies

$$
e_{+}^{+}=1, \quad e_{-}^{+}=0, \quad e_{I}^{+}=0,
$$

(where we decompose the local Lorentz indices as $m=(+,-, I)$ ), we shall have

$$
\Pi_{0}^{+}=1, \quad \Pi_{1}^{+}=0 .
$$

\footnotetext{
${ }^{2}$ Note that "rigid" here means that $\epsilon$ is a Killing spinor in the ten-dimensional type IIA target spacetime, hence determined by a finite number of constant parameters.
} 
Note that (17) is satisfied not only for a flat Minkowski background spacetime, but also for the pp-wave solution. Since the worldsheet metric is given by (13), we also deduce that in light-cone gauge we shall have

$$
\begin{array}{ll}
h_{00}=2 \Pi_{0}^{-}+\Pi_{0}^{I} \Pi_{0}^{I}, & h_{11}=\Pi_{1}^{I} \Pi_{1}^{I}, \quad h_{01}=\Pi_{1}^{-}+\Pi_{0}^{I} \Pi_{1}^{I}, \\
h_{11}=-h_{00}=\sqrt{-h}, & h_{01}=0 .
\end{array}
$$

Thus in particular we have

$$
\Pi_{0}^{-}=-\frac{1}{2}\left(\Pi_{0}^{I} \Pi_{0}^{I}+\Pi_{1}^{I} \Pi_{1}^{I}\right), \quad \Pi_{1}^{-}=-\Pi_{0}^{I} \Pi_{1}^{I}
$$

It is useful at this stage to introduce the two matrices

$$
P \equiv \Pi_{0}^{I} \Gamma_{I} \quad Q \equiv \Pi_{1}^{I} \Gamma_{I}
$$

in terms of which we have

$$
\Pi_{0}^{-}=-\frac{1}{2}\left(P^{2}+Q^{2}\right), \quad \Pi_{1}^{-}=-\frac{1}{2}(P Q+Q P)
$$

The matrix $\Gamma$ defined in (15) can then be written in the light-cone gauge as

$$
\Gamma=\frac{1}{\sqrt{-h}}\left(\frac{1}{2} \Gamma_{-} \Gamma_{+}(P Q+Q P)+\frac{1}{2} \Gamma_{-}\left(P Q P-Q^{3}\right)+\Gamma_{+} Q-Q P\right) \Gamma_{11},
$$

and we can write $1 / \sqrt{-h}$ as $Q^{-2}$.

To derive the residual worldsheet supersymmetry that emerges from light-cone gauge fixing of the kappa and rigid $\epsilon$ symmetries, we first note that we must preserve the light-cone condition $\Gamma^{+} \theta=0$ (i.e. $\Gamma_{-} \theta=0$ ), implying that

$$
\delta\left(\Gamma_{-} \theta\right)=\Gamma_{-}(\kappa+\epsilon)+\Gamma_{-} \Gamma \kappa=0 .
$$

From (23) this gives

$$
Q \Gamma_{-}(\kappa+\epsilon)+\left(\Gamma_{-} \Gamma_{+}+\Gamma_{-} P\right) \Gamma_{11} \kappa=0
$$

This can be viewed as an equation determining 16 of the 32 components of $\kappa$, in terms of $\epsilon$. Thus the $\kappa$ transformation is a compensating transformation that ensures the preservation of the light-cone condition $\Gamma_{-} \theta=0$ under rigid supersymmetry transformations. The residual symmetries will be given by $\delta\left(\Gamma_{+} \theta\right)=\Gamma_{+}(\kappa+\epsilon)+\Gamma_{+} \Gamma \kappa$. Using (23) and (25), we find after simple algebra that

$$
\delta\left(\Gamma_{+} \theta\right)=\frac{1}{2}\left(P+Q \Gamma_{11}\right) \Gamma_{+} \Gamma_{-} \epsilon+\Gamma_{+} \epsilon
$$


Note that $\kappa$ no longer appears; although one can solve for only 16 of the 32 components of $\kappa$ using (25), it is only these components that enter in the expression for $\delta\left(\Gamma_{+} \theta\right)$.

The residual transformations (26) comprise a standard type of homogeneous worldsheet supersymmetry, together with a shift symmetry. It is clearest to see this by choosing a $32=16 \otimes 2$ basis where

$$
\Gamma_{+}=\left(\begin{array}{cc}
0 & \sqrt{2} \\
0 & 0
\end{array}\right), \quad \Gamma_{-}=\left(\begin{array}{cc}
0 & 0 \\
\sqrt{2} & 0
\end{array}\right), \quad \Gamma_{I}=\left(\begin{array}{cc}
\gamma_{I} & 0 \\
0 & -\gamma_{I}
\end{array}\right), \quad \Gamma_{11}=\left(\begin{array}{cc}
\gamma_{9} & 0 \\
0 & -\gamma_{9}
\end{array}\right),
$$

where $\gamma_{9}=\prod_{I=1}^{8} \gamma_{I}, \Gamma_{ \pm}=\left(\Gamma_{9} \pm \Gamma_{0}\right) / \sqrt{2}$. One has

$$
\theta=\left(\begin{array}{l}
\theta_{1} \\
\theta_{2}
\end{array}\right), \quad \epsilon=\left(\begin{array}{c}
\epsilon_{1} \\
\epsilon_{2}
\end{array}\right), \quad \kappa=\left(\begin{array}{c}
\kappa_{1} \\
\kappa_{2}
\end{array}\right),
$$

and thus the gauge condition $\Gamma_{-} \theta=0$ implies $\theta_{1}=0$ while (26) gives

$$
\delta \theta_{2}=\frac{1}{\sqrt{2}}\left(p+q \gamma_{9}\right) \epsilon_{1}+\epsilon_{2}
$$

Here

$$
P=\left(\begin{array}{cc}
p & 0 \\
0 & -p
\end{array}\right), \quad Q=\left(\begin{array}{cc}
q & 0 \\
0 & -q
\end{array}\right) .
$$

Since $p=\Pi_{0}^{I} \gamma_{I}$ and $q=\Pi_{1}^{I} \gamma_{I}$, we see that $\epsilon_{1}$ describes a homogeneous (sometimes somewhat misleadingly called linearly-realised) supersymmetry, while $\epsilon_{2}$ describes an inhomogeneous shift symmetry.

The corresponding supersymmetry transformation of the bosonic coordinates in (14) then gives

$$
\begin{aligned}
\delta X^{I} & =\mathrm{i} \bar{\theta} \Gamma^{I}[(1+\Gamma) \kappa-\epsilon]+2 \mathrm{i} \bar{\theta} \Gamma^{I} \epsilon, \\
& =\frac{\mathrm{i}}{2} \bar{\theta} \Gamma^{I} \Gamma_{+}\left[\Gamma_{-}(1+\Gamma) \kappa-\Gamma_{-} \epsilon\right]+2 \mathrm{i} \bar{\theta} \Gamma^{I} \epsilon, \\
& =2 \mathrm{i} \bar{\theta} \Gamma^{I} \epsilon .
\end{aligned}
$$

One also easily checks that $\delta X^{+}=0$, and so the light-cone gauge condition $X^{+}=\tau$ is preserved. Thus in all we have

$$
\delta \theta_{2}=\frac{1}{\sqrt{2}}\left(p+q \gamma_{9}\right) \epsilon_{1}+\epsilon_{2}, \quad \delta X^{I}=2 \mathrm{i} \bar{\theta}_{2} \gamma^{I} \epsilon_{1}
$$

We see in particular that the "linearly-realised" worldsheet supersymmetries corresponding to $\epsilon_{1}$ are associated with Killing spinors $\epsilon$ that satisfy the projection condition

$$
\Gamma_{+} \epsilon=0
$$


Note that the 16 "ordinary" Killing spinors in the pp-wave background satisfy $\Gamma_{-} \epsilon=0$. Thus we see that the condition for having linearly-realised supersymmetry on the worldsheet precisely conflicts with the condition satisfied by the ordinary 16 Killing spinors in the ppwave. This means that none of the ordinary 16 Killing spinors gives rise to linearly-realised supersymmetry on the string worldsheet in lightcone gauge. By contrast, the supernumerary Killing spinors in a pp-wave solution, which are themselves subject to the opposite projection condition $\Gamma_{+} \epsilon=0$, are precisely compatible with the projection condition (33) for linearlyrealised worldsheet supersymmetries.

\section{Type IIB Green-Schwarz Action}

\subsection{Kappa symmetry and supersymmetry in type IIB}

The explicit component form of the Green-Schwarz action for the type IIB string on an arbitrary bosonic background was derived in [11], up to and including terms quadratic in the fermionic coordinates. The action was derived by explicitly implementing a T-duality transformation of the type IIA Green-Schwarz action in [11].

The type IIB Green-Schwarz action obtained in [11] was manipulated further in [8], where it was cast into a somewhat more convenient form. The notations and conventions of [11] and [8] have both been improved and updated in their latest hep-th versions. The reader is referred to these papers for background material and additional information on notation and conventions. The type IIB action as obtained in [8] takes the form

$$
\begin{aligned}
\mathcal{L}= & -\frac{1}{2} \sqrt{-h} h^{i j} \partial_{i} X^{\mu} \partial_{j} X^{\nu} g_{\mu \nu}+\frac{1}{2} \epsilon^{i j} \partial_{i} X^{\mu} \partial_{j} X^{\nu} B_{\mu \nu} \\
& +\mathrm{i} \partial_{i} X^{\mu} \bar{\theta} \gamma^{i j} \varrho_{0} \Gamma_{\mu} D_{j} \theta-\frac{\mathrm{i}}{8} \partial_{i} X^{\mu} \partial_{j} X^{\nu} \bar{\theta} \gamma^{i j} \varrho_{1} \Gamma_{\mu}{ }^{\rho \sigma} \theta G_{\nu \rho \sigma} \\
& +\frac{\mathrm{i}}{8} e^{\phi} \partial_{i} X^{\mu} \partial_{j} X^{\nu} \bar{\theta} \gamma^{i j} \Gamma_{\mu}\left[\Gamma^{\rho} \partial_{\rho} \chi+\frac{1}{6} \varrho_{2} \Gamma^{\rho_{1} \rho_{2} \rho_{3}} F_{\rho_{1} \rho_{2} \rho_{3}}+\frac{1}{240} \Gamma^{\rho_{1} \cdots \rho_{5}} F_{\rho_{1} \cdots \rho_{5}}\right] \Gamma_{\nu} \theta,
\end{aligned}
$$

where $G_{(3)}=d B_{(2)}$ is the NS-NS 3-form, $\phi$ and $\chi$ are the dilaton and axion, and $F_{(3)}$ and $F_{(5)}$ are the R-R 3-form and self-dual 5-form, and we have defined

$$
\gamma^{i j} \equiv \sqrt{-h} h^{i j}-\epsilon^{i j} \varrho_{2} .
$$

Hence, the conjugate $\bar{\theta}$ is defined by $\bar{\theta}=\theta^{\dagger} \Gamma_{0} \varrho_{0}$, and the "worldsheet" Dirac matrices are defined by $\varrho_{0}=-\mathrm{i} \tau_{2}, \varrho_{1}=\tau_{1}$, giving $\varrho_{2}=\tau_{3}$, where the $\tau_{a}$ are the usual Pauli matrices.

After some algebraic manipulations, we can show that the action following from (34) is invariant under local kappa transformations defined by

$$
\delta \theta=(1+\Gamma) \kappa, \quad \delta X^{\mu}=\mathrm{i} \bar{\theta} \Gamma^{\mu} \varrho_{0} \delta \theta,
$$


where the matrix $\Gamma$ is defined by

$$
\Gamma=\frac{1}{2 \sqrt{-h}} \epsilon^{i j} \partial_{i} X^{\mu} \partial_{j} X^{\nu} \Gamma_{\mu \nu} \varrho_{2} .
$$

(Note that to the quadratic order in fermions to which we are working, it suffices to replace the usual pulled-back supervielbeins $\Pi_{i}^{m} \equiv \partial_{i} Z^{M} E_{M}^{m}$ by $\partial_{i} X^{\mu} e_{\mu}^{m}$ in the expression for $\Gamma$, leading to (37).)

We can also show that the action following from (34) is invariant under rigid spacetime supersymmetry transformations

$$
\delta \theta=\epsilon, \quad \delta X^{\mu}=-\mathrm{i} \bar{\theta} \Gamma^{\mu} \varrho_{0} \epsilon,
$$

where $\epsilon$ is a Killing spinor in the bosonic background spacetime. Specifically, we can show that under (38), the Lagrangian (34) varies to give, up to total derivatives,

$$
\delta \mathcal{L}=2 \mathrm{i} \partial_{i} X^{\mu} \partial_{j} X^{\nu} \bar{\theta} \gamma^{i j} \varrho_{0} \Gamma_{\mu} \mathcal{D}_{\nu} \epsilon
$$

where

$$
\mathcal{D}_{\mu}=\nabla_{\mu}+\frac{1}{8} G_{\mu \rho \sigma} \varrho_{2} \Gamma^{\rho \sigma}-\frac{1}{8} e^{\phi}\left(\varrho_{0} \Gamma^{\sigma} \partial_{\sigma} \chi-\frac{1}{6} \varrho_{1} \Gamma^{\sigma_{1} \sigma_{2} \sigma_{3}} F_{\sigma_{1} \sigma_{2} \sigma_{3}}+\frac{1}{240} \varrho_{0} \Gamma^{\sigma_{1} \cdots \sigma_{5}} F_{\sigma_{1} \cdots \sigma_{5}}\right) \Gamma_{\mu} .
$$

Here $\nabla_{\mu} \equiv \partial_{\mu}+\frac{1}{4} \omega_{\mu}^{m n} \Gamma_{m n}$. Note that $\mathcal{D}_{\mu}$ is precisely the supercovariant derivative that appears in the spacetime supersymmetry transformation of the gravitini in type IIB supergravity, and so the Green-Schwarz action following from (34) is invariant under a rigid supersymmetry (38) where $\epsilon$ is a Killing spinor, satisfying $\mathcal{D}_{\mu} \epsilon=0$.

It is worth remarking that if we define

$$
\begin{aligned}
\widetilde{\mathcal{D}}_{i}= & D_{i}+\partial_{i} X^{\mu} \times \\
& {\left[\frac{1}{8} G_{\mu \rho \sigma} \varrho_{2} \Gamma^{\rho \sigma}-\frac{1}{8} e^{\phi}\left(\varrho_{0} \Gamma^{\sigma} \partial_{\sigma} \chi-\frac{1}{6} \varrho_{1} \Gamma^{\sigma_{1} \sigma_{2} \sigma_{3}} \Gamma_{\sigma_{1} \sigma_{2} \sigma_{3}}+\frac{1}{240} \varrho_{0} \Gamma^{\sigma_{1} \cdots \sigma_{5}} F_{\sigma_{1} \cdots \sigma_{5}}\right) \Gamma_{\mu}\right] }
\end{aligned}
$$

which when acting on a spinor function of the $X^{\mu}$ coordinates such as $\epsilon$ reduces to $\widetilde{\mathcal{D}}_{i} \epsilon=$ $\partial_{i} X^{\mu} \mathcal{D} \mu \epsilon$, we can write the Lagrangian (34) as

$$
\mathcal{L}=-\frac{1}{2} \sqrt{-h} h^{i j} \partial_{i} X^{\mu} \partial_{j} X^{\nu} g_{\mu \nu}+\frac{1}{2} \epsilon^{i j} \partial_{i} X^{\mu} \partial_{j} X^{\nu} B_{\mu \nu}+\mathrm{i} \partial_{i} X^{\mu} \bar{\theta} \gamma^{i j} \varrho_{0} \Gamma_{\mu} \widetilde{\mathcal{D}}_{j} \theta
$$

Thus the type IIB string action obtained in [11] and [8] does indeed satisfy this property, despite the doubts on this account that were raised in [14].

Note that if we define the doublet of Majorana-Weyl 32-component spinors

$$
\epsilon=\left(\begin{array}{l}
\epsilon_{1} \\
\epsilon_{2}
\end{array}\right)
$$


where the upper and lower components correspond to the positive and negative eigenstates of the worldsheet chirality matrix $\varrho_{2}$, and then define the complex Weyl spinor $\varepsilon \equiv \epsilon_{1}+\mathrm{i} \epsilon_{2}$, then we have the equivalences

$$
\varrho_{2}(\epsilon) \leftrightarrow \varepsilon^{*}, \quad \varrho_{0}(\epsilon) \leftrightarrow-\mathrm{i} \varepsilon, \quad \varrho_{1}(\epsilon) \leftrightarrow \mathrm{i} \varepsilon^{*}
$$

In terms of this complex Weyl notation, the gravitino transformation rule $\delta \psi_{\mu}=\mathcal{D}_{\mu} \epsilon$ with $\mathcal{D}_{\mu}$ given by (40) therefore becomes

$$
\begin{aligned}
\delta \psi_{\mu}= & \nabla_{\mu} \varepsilon+\frac{1}{8} G_{\mu \rho \sigma} \Gamma^{\rho \sigma} \varepsilon^{*} \\
& +\frac{i}{8} e^{\phi}\left(\Gamma^{\sigma} \Gamma_{\mu} \partial_{\sigma} \chi \varepsilon+\frac{1}{6} \Gamma^{\sigma_{1} \sigma_{2} \sigma_{3}} \Gamma_{\mu} F_{\sigma_{1} \sigma_{2} \sigma_{3}} \varepsilon^{*}+\frac{1}{240} \Gamma^{\sigma_{1} \cdots \sigma_{5}} \Gamma_{\mu} F_{\sigma_{1} \cdots \sigma_{5}} \varepsilon\right),
\end{aligned}
$$

where $\psi_{\mu}$ is now a complex Weyl spinor-vector, defined analogously to $\varepsilon$.

\subsection{Type IIB worldsheet supersymmetry in the light-cone gauge}

The treatment of kappa symmetry and supersymmetry for the type IIB Green-Schwarz action in light-cone gauge closely parallels the type IIA discussion in section 2.2, so we shall just summarise the key results. The essential change is that the matrix $\Gamma$ appearing in the kappa transformation rule (36) is now given by

$$
\Gamma=\frac{1}{2 \sqrt{-h}} \epsilon^{i j} \Pi_{i}^{m} \Pi_{j}^{m} \Gamma_{m n} \varrho_{2}
$$

rather than (37). Following the same steps as in section 2.2, we impose the light-cone condition $\Gamma_{-} \theta=0$, and so the compensating kappa transformation that maintains this gauge, analogous to $(25)$, is

$$
Q \Gamma_{-}(\kappa+\epsilon)+\left(\Gamma_{-} \Gamma_{+}+\Gamma_{-} P\right) \varrho_{2} \kappa=0 .
$$

We then find that the residual transformations of $\Gamma_{+} \theta$, analogous to the type IIA result (26), are given by

$$
\delta\left(\Gamma_{+} \theta\right)=\frac{1}{2}\left(P+Q \varrho_{2}\right) \Gamma_{+} \Gamma_{-} \epsilon+\Gamma_{+} \epsilon
$$

As in the type IIA case, we can see that the residual linearly-realised supersymmetries are parametrised by Killing spinors $\epsilon$ that are subject to the projection condition

$$
\Gamma_{+} \epsilon=0
$$

whilst the remaining Killing spinors subject to the opposite projection condition $\Gamma_{-} \epsilon=0$ correspond to inhomogeneous shift transformations. Thus we see that here for type IIB 
we have the same result as in type IIA, namely that the 16 ordinary Killing spinors in any pp-wave solution, which are subject to the projection $\Gamma_{-} \epsilon=0$, are associated with inhomogeneous shift symmetries on the string worldsheet. By contrast, any supernumerary Killing spinors, which are subject to the projection condition $\Gamma_{+} \epsilon=0$, are associated with linearly-realised worldsheet supersymmetries.

\section{Worldsheet Supersymmetry in pp-wave Backgrounds}

\subsection{Killing spinors in pp-wave backgrounds}

Before considering pp-waves of the type that arise in Penrose limits and their generalisations, it is helpful to begin by recalling the situation for purely gravitational wave solutions in supergravities, where only the metric itself takes a non-trivial form. The solutions of this type that we shall consider are given by

$$
d s^{2}=2 d x^{+} d x^{-}+K d x^{+^{2}}+d z_{i}^{2}
$$

where $K$ is a function of the $z_{i}$. ( $K$ could also be allowed to depend on $x^{+}$, but we shall not consider this here.) The condition for the Ricci tensor to vanish is that

$$
\square K=0,
$$

where $\square$ is the Laplacian $\partial_{i}^{2}$ on the flat transverse space whose coordinates are the $z_{i}$.

If this Ricci-flat solution is taken in the context of a supergravity theory, the supersymmetry transformation rules will imply that the background is supersymmetric for spinor parameters $\epsilon$ that satisfy $\nabla \epsilon=0$, where $\nabla \equiv d+\frac{1}{4} \omega^{A B} \Gamma_{A B}$ is the Lorentz-covariant exterior derivative. It is easily seen that in the background (50), this derivative is given by

$$
\nabla=d+\frac{1}{4} K_{i} d x^{+} \Gamma_{-i}
$$

where $K_{i} \equiv \partial_{i} K$. A Killing spinor $\epsilon$ satisfies $\partial_{+} \epsilon+\frac{1}{4} K_{i} \Gamma_{-i} \epsilon=0, \partial_{-} \epsilon=0$ and $\partial_{i} \epsilon=0$, from which it is straightforward to show that $\epsilon$ must be constant, and satisfy the projection condition

$$
\Gamma_{-} \epsilon=0
$$

The conclusion from the above discussion is that whenever we have a purely gravitational wave solution in supergravity, there will be Killing spinors whose total number is precisely one half of the number that would arise in a flat background, on account of the projection 
condition (53). This counting of Killing spinors is independent of the specific details of the harmonic function $K$.

In the pp-wave solutions that have been considered recently, arising as Penrose limits of $\mathrm{AdS} \times$ Sphere solutions in supergravity, there are additional non-trivial contributions to the bosonic background, aside from the gravitational wave metric (50) itself. In fact in all cases, the extra fields involved in the recently-considered pp-wave solutions take the form of constant background values for antisymmetric tensor field strengths in the supergravity theory. For a $p$-index field strength $F_{p}$, this background has the structure

$$
F_{p}=d x^{+} \wedge \Phi_{p-1}
$$

where $\Phi_{p-1}$ is a constant $(p-1)$-form in the transverse space. The previous homogeneous equation (51) for $K$ now becomes

$$
\square K=-k\left|\Phi_{p-1}\right|^{2}
$$

where $k$ is a constant.

In the transformation rules for the gravitini and spin- $\frac{1}{2}$ fields in the supergravity theory, these field strengths give contributions of the form

$$
\begin{aligned}
\delta \psi_{\mu} & =\nabla_{\mu} \epsilon+c_{1} F_{\mu \nu_{1} \cdots \nu_{p-1}} \Gamma^{\nu_{1} \cdots \nu_{p-1}} \epsilon+c_{2} F^{\nu_{1} \cdots \nu_{p}} \Gamma_{\mu \nu_{1} \cdots \nu_{p}} \epsilon+\cdots \\
\delta \chi & =c_{3} F_{\nu_{1} \cdots \nu_{p}} \Gamma^{\nu_{1} \cdots \nu_{p}} \epsilon+\cdots .
\end{aligned}
$$

It is clear that the contribution of a field strength (54) in the supersymmetry transformation rules for $\delta \psi_{-}, \delta \psi_{i}$ and $\delta \chi$ will all involve a $\Gamma_{-}$projection acting on $\epsilon$, and thus these terms will all vanish if $\epsilon$ again satisfies the projection condition (53). There can be a non-vanishing contribution only in $\delta \psi_{+}$, which means that the previous equation $\partial_{+} \epsilon=0$ in the purely gravitational case now becomes an equation determining the $x^{+}$dependence of $\epsilon$. The upshot is that all the Killing spinors in the previous purely gravitational wave background will survive, possibly now with $x^{+}$dependence, in the more general solutions with constant field strengths. In other words, in the new pp-wave solutions there will always exist Killing spinors satisfying the projection condition (53), whose number is again precisely one half of the number of Killing spinors for a flat background. In papers $[7,8]$, the Killing spinors that exist for arbitrary choices of $K$ solving the bosonic equation (55) were referred to as Standard Killing Spinors.

In the recently-considered pp-wave solutions, there can exist further Killing spinors over and above the standard ones. These can arise only if the constant form $\Phi_{p-1}$ and the solution 
to (55) for $K$ are chosen to have a very special form. In particular, $K$ must be taken to be a specific purely quadratic function of the transverse coordinates $z_{i}$. By contrast to the standard Killing spinors described above, these additional Killing spinors, if they occur, are subject to the opposite projection condition, namely

$$
\Gamma_{+} \epsilon=0
$$

These Killing spinors were referred to as Supernumerary Killing Spinors in [7, 8].

To summarise, if we consider pp-waves in a supergravity theory that has $N$ supercharges, there will always exist $\frac{1}{2} N$ standard Killing spinors, which satisfy the projection condition (53). There may in addition exist a number $N_{\text {sup }}$ of supernumerary Killing spinors, with

$0 \leq N_{\text {sup }} \leq \frac{1}{2} N$, which satisfy the opposite (and orthogonal) projection condition (57). The number that occur depends on the details of the constant form $\Phi_{p-1}$, and the choice of the quadratic function $K$.

\subsection{Linearly-realised worldsheet supersymmetries}

We saw in sections (2.2) and (3.2) that when one imposes the light-cone gauge conditions

$$
X^{+}=\tau, \quad \sqrt{-h} h^{i j}=\eta^{i j} \quad \Gamma_{-} \theta=0
$$

on the covariant Green-Schwarz type IIA or IIB actions, the local kappa symmetry and rigid spacetime supersymmetry transmute into a rigid worldsheet supersymmetry of the light-cone string action. To be more precise, we saw that there is an $N=1$ linearly-realised worldsheet supersymmetry corresponding to every Killing spinor in the ten-dimensional target spacetime that satisfies the projection condition

$$
\Gamma_{+} \epsilon=0
$$

In view of the discussion of Killing spinors in pp-wave backgrounds given in $[7,8]$, which was summarised in section (4.1) above, we see therefore that the 16 standard Killing spinors in any type IIA or type IIB pp-wave background will never give rise to linearly-realised worldsheet supersymmetries, since they satisfy instead the orthogonal projection condition (53). By contrast, every supernumerary Killing spinor, since it satisfies the projection condition (57), will give rise to a linearly-realised worldsheet supersymmetry.

This result was in fact foreseen in $[7,8]$. The argument used there was based on the observation that in the light-cone string action the masses of the bosonic fields $X^{I}$ and the fermionic fields $\theta$ are in general unrelated, in a pp-wave background. It is manifest 
that if the boson and fermion masses are unequal then there cannot be any linearly-realised worldsheet supersymmetry. Only in the case of special pp-wave backgrounds where there exist supernumerary Killing spinors does one find that there is a precise matching of boson and fermion masses. This provided circumstantial evidence in $[7,8]$ for the connection between supernumerary Killing spinors and linearly-realised worldsheet supersymmetries, which we have now made precise in this paper.

\section{Green-Schwarz Actions for pp-waves in Physical Gauge}

\subsection{Type IIA worldsheet supersymmetries in the physical gauge}

Let us contrast the light-cone analysis given above with what happens if we instead choose a physical gauge for the type IIA Green-Schwarz action. This gauge can be taken to be

$$
X^{0}=\tau, \quad X^{9}=\sigma, \quad\left(1+\Gamma_{*}\right) \theta=0,
$$

where the matrix $\Gamma_{*}$ is defined by

$$
\Gamma_{*} \equiv \Gamma_{1} \cdots \Gamma_{8}
$$

i.e. it is the product of the eight transverse-space Dirac matrices. This fermionic gauge condition is motivated by considering the form of the matrix $\Gamma$ in the field-independent static limit in the bosonic part of the physical gauge, where all the coordinates $X^{\mu}$ except for those set equal to the worldsheet coordinates vanish. From (15) we see that $\Gamma$ then becomes just $\Gamma_{09} \Gamma_{11}$, which is precisely $\Gamma_{*}$. Thus the leading-order form of the kappa symmetry transformation is $\delta \theta=\left(1+\Gamma_{*}\right) \kappa$, and so we can expect to be able to use this symmetry to $\operatorname{set}^{3}\left(1+\Gamma_{*}\right) \theta=0$. In fact we shall show in section 6.4 that this physicalgauge fixing condition is precisely what arises naturally in a string/pp-wave intersecting supergravity solution.

We now find it helpful to decompose the Dirac $32 \times 32$ matrices as ${ }^{4}$

$$
\Gamma_{0}=\left(\begin{array}{cccc}
0 & 1 & 0 & 0 \\
-1 & 0 & 0 & 0 \\
0 & 0 & 0 & 1 \\
0 & 0 & -1 & 0
\end{array}\right), \quad \Gamma_{9}=\left(\begin{array}{cccc}
0 & 1 & 0 & 0 \\
1 & 0 & 0 & 0 \\
0 & 0 & 0 & 1 \\
0 & 0 & 1 & 0
\end{array}\right), \quad \Gamma_{I}=\left(\begin{array}{cccc}
0 & 0 & \beta_{I} & 0 \\
0 & 0 & 0 & -\beta_{I} \\
\tilde{\beta}_{I} & 0 & 0 & 0 \\
0 & -\tilde{\beta}_{I} & 0 & 0
\end{array}\right)
$$

\footnotetext{
${ }^{3}$ One might instead use the kappa transformation to set $(1+\Gamma) \theta=0$ as a gauge condition, but this would be less convenient since it is field dependent.

${ }^{4}$ Each entry is an $8 \times 8$ matrix here.
} 
where $\beta_{I}=\left(\beta_{I^{\prime}}, \beta_{8}\right)=\left(\tilde{\gamma}_{I^{\prime}}, \mathrm{i}\right)$ and $\tilde{\beta}_{I}=\left(\tilde{\beta}_{I^{\prime}}, \tilde{\beta}_{8}\right)=\left(\tilde{\gamma}_{I^{\prime}},-\mathrm{i}\right)$ are the Van der Waerden symbols of eight dimensions, with $\tilde{\gamma}_{I^{\prime}}$ being the $8 \times 8$ Dirac matrices in seven dimensions. With these conventions, we have

$$
\Gamma_{*}=\left(\begin{array}{cccc}
1 & 0 & 0 & 0 \\
0 & 1 & 0 & 0 \\
0 & 0 & -1 & 0 \\
0 & 0 & 0 & -1
\end{array}\right), \quad \Gamma_{11}=\left(\begin{array}{cccc}
1 & 0 & 0 & 0 \\
0 & -1 & 0 & 0 \\
0 & 0 & -1 & 0 \\
0 & 0 & 0 & 1
\end{array}\right) .
$$

The matrix $\Gamma$ that appears in the kappa transformations can be written as

$$
\Gamma=\frac{1}{2} M^{m n} \Gamma_{m n} \Gamma_{11}=-\mathbb{1}+A+B
$$

where

$$
\begin{aligned}
& A \equiv \mathbb{1}+M^{09} \Gamma_{09} \Gamma_{11}+\frac{1}{2} M^{I J} \Gamma_{I J} \Gamma_{11} \\
& B \equiv=M^{0 I} \Gamma_{0 I} \Gamma_{11}+M^{I 9} \Gamma_{I 9} \Gamma_{11}
\end{aligned}
$$

This splitting of the terms in $\Gamma$ is chosen so that

$$
\left[A, \Gamma_{*}\right]=0, \quad\left\{B, \Gamma_{*}\right\}=0 .
$$

The quantities $M^{m n}$ are given by

$$
\begin{aligned}
M^{09} & =\frac{1}{\sqrt{-h}}\left(\Pi_{0}^{0} \Pi_{1}^{9}-\Pi_{1}^{0} \Pi_{0}^{9}\right), \\
M^{0 I} & =\frac{1}{\sqrt{-h}}\left(\Pi_{0}^{0} \Pi_{1}^{I}-\Pi_{1}^{0} \Pi_{0}^{I}\right), \\
M^{I 9} & =\frac{1}{\sqrt{-h}}\left(\Pi_{0}^{I} \Pi_{1}^{9}-\Pi_{1}^{I} \Pi_{0}^{9}\right), \\
M^{I J} & =\frac{1}{\sqrt{-h}}\left(\Pi_{0}^{I} \Pi_{1}^{J}-\Pi_{1}^{I} \Pi_{0}^{J}\right) .
\end{aligned}
$$

We now proceed with the physical gauge fixing. Requiring that $\left(1+\Gamma_{*}\right) \theta=0$ be preserved under combined supersymmetry and kappa transformations implies

$$
A\left(1+\Gamma_{*}\right) \kappa+B\left(1-\Gamma_{*}\right) \kappa+\left(1+\Gamma_{*}\right) \epsilon=0
$$

and then the transformation of $\left(1-\Gamma_{*}\right) \theta$ is given by

$$
\left(1-\Gamma_{*}\right) \delta \theta=A\left(1-\Gamma_{*}\right) \kappa+B\left(1+\Gamma_{*}\right) \kappa+\left(1-\Gamma_{*}\right) \epsilon
$$

The commutation properties of $A$ and $B$ given in (66) imply that we can write them in $16 \times 16$ block-matrix form, for which $\Gamma_{*}$ in $(63)$ becomes

$$
\Gamma_{*}=\left(\begin{array}{cc}
\mathbb{1}_{16} & 0 \\
0 & -\mathbb{1}_{16}
\end{array}\right),
$$


as

$$
A=\left(\begin{array}{cc}
a & 0 \\
0 & \tilde{a}
\end{array}\right), \quad B=\left(\begin{array}{cc}
0 & b \\
\tilde{b} & 0
\end{array}\right)
$$

Defining

$$
\theta=\left(\begin{array}{l}
\theta_{1} \\
\theta_{2}
\end{array}\right), \quad \kappa=\left(\begin{array}{l}
\kappa_{1} \\
\kappa_{2}
\end{array}\right), \quad \epsilon=\left(\begin{array}{c}
\epsilon_{1} \\
\epsilon_{2}
\end{array}\right),
$$

where the upper and lower 16 components are the projections under $\frac{1}{2}\left(1 \pm \Gamma_{*}\right)$ with $\Gamma_{*}$ given in (70), we therefore find that (68) and (69) become

$$
\begin{aligned}
0 & =a \kappa_{1}+b \kappa_{2}+\epsilon_{1}, \\
\delta \theta_{2} & =\tilde{a} \kappa_{2}+\tilde{b} \kappa_{0}+\epsilon_{2} .
\end{aligned}
$$

Note that the physical-gauge choice means that $\Pi_{0}^{0}$ and $\Pi_{1}^{9}$ are of order 1 , whilst all the other $\Pi_{i}^{m}$ are of first order in physical fields, plus higher terms. This means that up to linear order in coordinates, expanding around the static background $X^{0}=\tau, X^{9}=\sigma, X^{I}=$ $0, \theta=0$, we have

$$
M_{09}=1, \quad M^{0 I}=\partial_{1} X^{I}, \quad M^{I 9}=\partial_{0} X^{I}, \quad M^{I J}=0 .
$$

In particular, we note that the matrix $A$ has the form

$$
A=\left(\begin{array}{llll}
2 & 0 & 0 & 0 \\
0 & 2 & 0 & 0 \\
0 & 0 & 0 & 0 \\
0 & 0 & 0 & 0
\end{array}\right)+\text { linear terms }
$$

which means that its upper $16 \times 16$ block denoted by $a$ in $(71)$ is invertible. Thus we can solve the first equation in (73) for $\kappa_{1}$, and substitute into the second. This gives

$$
\delta \theta_{2}=-\tilde{b} a^{-1} \epsilon_{1}+\epsilon_{2}+\left(\tilde{a}-\tilde{b} a^{-1} b\right) \kappa_{2} .
$$

Next, we note from the fact that $\Gamma^{2}=1$ that we shall have $(A+B)(A+B-2)=0$, which from (71) gives

$$
a(a-2)+b \tilde{b}=0, \quad a b+b(\tilde{a}-2)=0, \quad \tilde{b}(a-2)+\tilde{a} \tilde{b}=0, \quad \tilde{b} b+\tilde{a}(\tilde{a}-2)=0 .
$$

Multiplying the second equation by $\tilde{b} a^{-1}$ from the left, and subtracting the fourth, whilst noting that $\tilde{a}$ is of first order in fields and that therefore $(\tilde{a}-2)$ is invertible, we deduce that $\tilde{a}=\tilde{b} a^{-1} b$. This shows that $\kappa_{2}$ drops out completely from (76), and hence that we have

$$
\delta \theta_{2}=-\tilde{b} a^{-1} \epsilon_{1}+\epsilon_{2}
$$


Finally, we can see from the previous definitions that up to linear order in fields (for which, since $\tilde{b}$ is of linear order we can take $a^{-1}$ in (78) to be $\frac{1}{2}$ ),

$$
\delta \theta_{2}=-\frac{1}{2} \tilde{b} \epsilon_{1}+\epsilon_{2}
$$

where

$$
\tilde{b}=\left(\begin{array}{cc}
0 & \tilde{\beta}_{I} \partial_{-} X^{I} \\
-\tilde{\beta}_{I} \partial_{+} X^{I} & 0
\end{array}\right) .
$$

Here, we have defined the worldsheet derivatives $\partial_{ \pm}=\partial_{1} \pm \partial_{0}$. We see, therefore, that $\epsilon_{1}$ parametrises linearly-realised supersymmetries, while $\epsilon_{2}$ describes an inhomogeneous shift symmetry. In summary, therefore, we have shown that in the physical gauge the parameter $\epsilon$ of the linearly-realised worldsheet supersymmetry is subject to the projection condition

$$
\left(1-\Gamma_{*}\right) \epsilon=0
$$

The projection condition (81) for linearly-realised supersymmetries in the physical gauge is very different from the analogous projection condition (33) that we obtained in the lightcone gauge. In particular, we find that whereas the 16 standard Killing spinors in any ppwave background are all incompatible with the light-cone projection (33), in the physical gauge some of the standard Killing spinors are compatible with the projection (81). In fact, as we shall see in detail in section 6 , there are both standard and supernumerary Killing spinors in pp-waves that are compatible with the projection (81) in the physical gauge. This means that in the physical gauge, there can be linearly-realised worldsheet supersymmetries even in a pp-wave background that has only the 16 standard Killing spinors.

\subsection{Type IIB worldsheet supersymmetries in the physical gauge}

The imposition of a physical gauge condition in the type IIB string proceeds very analogously to the discussion for type IIA in section 5.1. Again, one can determine a suitable choice of fermionic gauge condition by starting with a static string configuration where $X^{0}=\tau, X^{9}=\sigma$ and all other bosonic coordinates vanish. The full expression

$$
\Gamma=\frac{1}{2 \sqrt{-h}} \epsilon^{i j} \Pi_{i}^{m} \Pi_{j}^{n} \Gamma_{m n} \varrho_{2} .
$$

then reduces to

$$
\Gamma \longrightarrow \Gamma_{\#} \equiv \Gamma_{09} \varrho_{2}
$$

and so we are led to impose

$$
X^{0}=\tau, \quad X^{9} \equiv \sigma, \quad\left(1+\Gamma_{\#}\right) \theta=0
$$


as the physical gauge condition. We shall show later that this fermionic projection agrees precisely with the one that is encountered from the string component in a type IIB string/ppwave intersection.

As in the type IIA discussion in section 5.1, it is convenient to make a choice of Dirac matrix basis that is adapted to the gauge condition. Specifically, we choose a basis where $\Gamma_{\#}$ is diagonal, implying that its non-vanishing components will then be \pm 1 , and with the positive components appearing in the upper left-hand part of the matrix. We can start from the basis (62), and first perform a similarity transformation in which $\Gamma_{11}$ becomes

$$
\Gamma_{11}=\left(\begin{array}{cccc}
1 & 0 & 0 & 0 \\
0 & 1 & 0 & 0 \\
0 & 0 & -1 & 0 \\
0 & 0 & 0 & -1
\end{array}\right)
$$

This can be achieved by exchanging the roles of the second and fourth rows and columns, leading to

$$
\Gamma_{0}=\left(\begin{array}{cccc}
0 & 0 & 0 & 1 \\
0 & 0 & -1 & 0 \\
0 & 1 & 0 & 0 \\
-1 & 0 & 0 & 0
\end{array}\right), \quad \Gamma_{9}=\left(\begin{array}{cccc}
0 & 0 & 0 & 1 \\
0 & 0 & 1 & 0 \\
0 & 1 & 0 & 0 \\
1 & 0 & 0 & 0
\end{array}\right), \quad \Gamma_{I}=\left(\begin{array}{cccc}
0 & 0 & \beta_{I} & 0 \\
0 & 0 & 0 & -\tilde{\beta}_{I} \\
\tilde{\beta}_{I} & 0 & 0 & 0 \\
0 & -\beta_{I} & 0 & 0
\end{array}\right)
$$

Note that the Dirac matrix combinations $\Gamma_{m n}$ that appear in $\Gamma$ all have components only in the upper left and lower right $16 \times 16$ blocks.

The spinors $\theta$ in type IIB are chiral, and so only the upper 16 components in the new basis will be non-vanishing. We can thus focus attention on these components, and now consider the resulting $32 \times 32$ matrices in the tensor product with the worldsheet Dirac matrices $\varrho_{a}$. In particular, with $\varrho_{2}=\tau_{3}$ we shall have

$$
\begin{gathered}
\Gamma_{\#}=\Gamma_{09} \varrho_{2} \longrightarrow\left(\begin{array}{cccc}
1 & 0 & 0 & 0 \\
0 & -1 & 0 & 0 \\
0 & 0 & -1 & 0 \\
0 & 0 & 0 & 1
\end{array}\right), \Gamma_{0 I} \varrho_{2} \longrightarrow\left(\begin{array}{cccc}
0 & -\beta_{I} & 0 & 0 \\
-\tilde{\beta}_{I} & 0 & 0 & 0 \\
0 & 0 & 0 & \beta_{I} \\
0 & 0 & \tilde{\beta}_{I} & 0
\end{array}\right), \quad(87) \\
\Gamma_{I 9} \varrho_{2} \longrightarrow\left(\begin{array}{cccc}
0 & \beta_{I} & 0 & 0 \\
-\tilde{\beta}_{I} & 0 & 0 & 0 \\
0 & 0 & 0 & -\beta_{I} \\
0 & 0 & \tilde{\beta}_{I} & 0
\end{array}\right), \Gamma_{I J} \varrho_{2} \longrightarrow\left(\begin{array}{cccc}
\beta_{I} \tilde{\beta}_{J} & 0 & 0 & 0 \\
0 & \tilde{\beta}_{I} \beta_{J} & 0 & 0 \\
0 & 0 & -\beta_{I} \tilde{\beta}_{J} & 0 \\
0 & 0 & 0 & -\tilde{\beta}_{I} \beta_{J}
\end{array}\right) .
\end{gathered}
$$

Finally, we apply a similarity transformation whose effect is to exchange the roles of the 
second and fourth rows and columns in these matrices, leading to

$$
\begin{gathered}
\Gamma_{\#}=\Gamma_{09} \varrho_{2} \longrightarrow\left(\begin{array}{cccc}
1 & 0 & 0 & 0 \\
0 & 1 & 0 & 0 \\
0 & 0 & -1 & 0 \\
0 & 0 & 0 & -1
\end{array}\right), \Gamma_{0 I} \varrho_{2} \longrightarrow\left(\begin{array}{cccc}
0 & 0 & 0 & -\beta_{I} \\
0 & 0 & \tilde{\beta}_{I} & 0 \\
0 & \beta_{I} & 0 & 0 \\
-\tilde{\beta}_{I} & 0 & 0 & 0
\end{array}\right), \quad(88) \\
\Gamma_{I 9} \varrho_{2} \longrightarrow\left(\begin{array}{cccc}
0 & 0 & 0 & \beta_{I} \\
0 & 0 & \tilde{\beta}_{I} & 0 \\
0 & -\beta_{I} & 0 & 0 \\
-\tilde{\beta}_{I} & 0 & 0 & 0
\end{array}\right), \Gamma_{I J} \varrho_{2} \longrightarrow\left(\begin{array}{cccc}
\beta_{I} \tilde{\beta}_{J} & 0 & 0 & 0 \\
0 & -\tilde{\beta}_{I} \beta_{J} & 0 & 0 \\
0 & 0 & -\beta_{I} \tilde{\beta}_{J} & 0 \\
0 & 0 & 0 & \tilde{\beta}_{I} \beta_{J}
\end{array}\right) .
\end{gathered}
$$

In this basis, when we construct $\Gamma \equiv-\mathbb{1}+A+B$ with

$$
\begin{aligned}
A & \equiv \mathbb{1}+M^{09} \Gamma_{09} \varrho_{2}+\frac{1}{2} M^{I J} \Gamma_{I J} \varrho_{2}, \\
B & \equiv M^{0 I} \Gamma_{0 I} \varrho_{2}+M^{I 9} \Gamma_{I 9} \varrho_{2},
\end{aligned}
$$

the fact that $\left[A, \Gamma_{\#}\right]=0$ and $\left\{B, \Gamma_{\#}\right\}=0$ implies that in $16 \times 16$ block form we shall have

$$
A=\left(\begin{array}{cc}
a & 0 \\
0 & \tilde{a}
\end{array}\right), \quad B=\left(\begin{array}{cc}
0 & b \\
\tilde{b} & 0
\end{array}\right) .
$$

This is closely parallel to the situation for the type IIA string, although here the $16 \times 16$ matrices $(a, \tilde{a}, b, \tilde{b})$ are different from those appearing in (71).

From this point on, the imposition of the physical gauge conditions proceeds exactly in parallel with the type IIA discussion in section 5.1. Thus we impose $\left(1+\Gamma_{\#}\right) \theta=0$, solve for the compensating kappa transformation that preserves this gauge, and thereby arrive at the residual transformations

$$
\delta \theta_{2}=-\tilde{b} a^{-1} \epsilon_{1}+\epsilon_{2}
$$

where we have written

$$
\theta=\left(\begin{array}{l}
\theta_{1} \\
\theta_{2}
\end{array}\right), \quad \epsilon=\left(\begin{array}{c}
\epsilon_{1} \\
\epsilon_{2}
\end{array}\right) .
$$

To first order in fields around the static string configuration, we shall therefore have

$$
\delta \theta_{2}=-\frac{1}{2} \tilde{b} \epsilon_{1}+\epsilon_{2}
$$

with

$$
\tilde{b}=\left(\begin{array}{cc}
0 & \beta_{I} \partial_{-} X^{I} \\
-\tilde{\beta}_{I} \partial_{+} X^{I} & 0
\end{array}\right) .
$$

The linearly-realised supersymmetries are parametrised by $\epsilon_{1}$, i.e. by Killing spinors $\epsilon$ that are subject to the projection condition

$$
\left(1-\Gamma_{\#}\right) \epsilon=0
$$


As in the case of type IIA strings, so here for type IIB strings we see that the projection condition (95) for linearly-realised worldsheet supersymmetries in the physical gauge is very different from the analogous projection (49) in the light-cone gauge. Again, this implies that both standard as well as supernumerary Killing spinors in a pp-wave solution can give rise to linearly-realised worldsheet supersymmetries in the physical gauge. This is discussed in more detail in section 6 .

\subsection{Absence of mass-terms in the physical gauge}

The forms of the complete Green-Schwarz actions, after imposing the physical gauge conditions, are quite complicated and we shall not present them in detail here. Instead, we shall focus just on the sectors where mass terms for the bosons and fermions might arise, in order to demonstrate that they are in fact absent.

First, we consider the bosonic sector, and consider the term

$$
\mathcal{L}_{0}=-\frac{1}{2} \sqrt{-h} h^{i j} \partial_{i} X^{\mu} \partial_{j} X^{\nu}
$$

which is in fact common to both the type IIA and the type IIB Green-Schwarz actions. Looking at this sector will be sufficient to demonstrate the absence of mass terms for the transverse bosonic coordinates $X^{I}$ in a pp-wave background.

In this bosonic sector, we have $h_{i j}=\partial_{i} X^{\mu} \partial_{j} X^{\nu} g_{\mu \nu}$, and the metric in the pp-wave background is given in (50). The Lagrangian (96), which can be written simply as $\mathcal{L}_{0}=$ $-\sqrt{-h}$, is therefore given by

$$
\begin{aligned}
\mathcal{L}_{0} & =-\sqrt{1+2 \partial_{+} X^{I} \partial_{-} X^{I}-K \partial_{-} X^{I} \partial_{+} X^{I}+\left(\partial_{+} X^{I} \partial_{-} X^{I}\right)^{2}-\left(\partial_{-} X^{I}\right)^{2}\left(\partial_{+} X^{J}\right)^{2}}, \\
& =-1-\partial_{+} X^{I} \partial_{-} X^{I}+\text { interaction terms } .
\end{aligned}
$$

In particular, we see that, unlike in the light-cone gauge, there are no mass terms for the $X^{I}$ transverse coordinates.

For the fermions, we note that in both the type IIA and type IIB Green-Schwarz actions, the source of the fermion masses in the light-cone analysis is the relevant R-R coupling term, which has the form

$$
\mathcal{L}_{\mathrm{RR}}=c e^{\phi} \partial_{i} X^{\mu} \partial_{j} X^{\nu} \bar{\theta} \alpha^{i j} \Gamma_{\mu} \Gamma^{\rho_{1} \cdots \rho_{p}} F_{\rho_{1} \cdots \rho_{p}} \Gamma_{\nu} \theta
$$

where $\alpha^{i j}$ represents either $\beta^{i j}$ in the type IIA case, given in (1) (possibly with an additional $\Gamma_{11}$ factor), or $\gamma^{i j}$ in the type IIB case, given in (34) (possibly with an extra factor of $\varrho_{2}$ ). In all cases, the relevant R-R field in the pp-wave solution has the form $F=\mu d x^{+} \wedge \Phi$. 
In the physical gauge, purely quadratic fermion terms will arise when $\partial_{i} X^{\mu}$ and $\partial_{j} X^{\nu}$ have their constant background contributions coming from $\partial_{+} X^{+}=1$ and $\partial_{-} X^{-}=1$, and so we see that the matrix between the $\bar{\theta}$ and $\theta$ fields in (98) will involve

$$
\Gamma_{+}\left(\Gamma_{-} W\right) \Gamma_{-} \quad \text { or } \quad \Gamma_{-}\left(\Gamma_{-} W\right) \Gamma_{+},
$$

where $W=\frac{1}{p !} \Gamma^{i_{1} \cdots i_{p}} \Phi_{i_{1} \cdots i_{p}}$. However, both these terms vanish, because $\Gamma_{-} \Gamma_{-}=0$, and so we see that in the physical gauge, there are no fermion mass terms.

We showed in sections 5.1 and 5.2 that there will always be linearly-realised worldsheet supersymmetries for pp-wave backgrounds in the physical gauge, even when there are only the 16 "standard" Killing spinors and no supernumerary Killing spinors. It is satisfactory, therefore, that we have found that there are no mass terms for either the bosons or the fermions in the physical gauge, since this is compatible with the linearly-realised supersymmetry.

\section{Strings in pp-wave Backgrounds: Supergravity Solutions}

In this section, we look for solutions in type IIA and type IIB supergravity corresponding to fundamental strings in the background of pp-waves. These solutions can be viewed as classical supergravity realisations of a string action with a pp-wave target spacetime background. As discussed in section 5, the supergravity solutions describing strings in pp-wave backgrounds will correspond to string actions that are expressed in the physical gauge.

The ten-dimensional metrics describing solutions for strings in pp-wave backgrounds have the same general structure as one finds for intersecting $p$-brane solutions. Thus the metric is given by

$$
d s_{10}^{2}=H^{-3 / 4}\left(2 d x^{+} d x^{-}+K d x^{+^{2}}\right)+H^{1 / 4} d z_{i}^{2},
$$

where $H$ and $K$ are taken to depend only on the eight transverse coordinates $z^{i}$. In a standard string/wave intersection, which could be either in the type IIA or type IIB theory, $H$ and $K$ would both be harmonic functions and the string source would be provided by the NS-NS 3-form field strength. The 3-form and dilaton would be given by

$$
F_{(3)}=d H^{-1} \wedge d x^{+} \wedge d x^{-}, \quad \phi=-\frac{1}{2} \log H .
$$

For our purposes, we now need to introduce additional form-field fluxes, just as in the pure pp-wave solutions. It is not a priori obvious that we can still find "intersecting" solutions, 
but in fact, as we shall show below, we are able to find examples both in type IIA and type IIB supergravity.

Before moving to the specific discussions for the type IIA and type IIB supergravities, it is useful to collect some general results that are common to the two cases.

We introduce the following vielbein 1-forms for the ten-dimensional metric (100):

$$
e^{+}=H^{-3 / 8} d x^{+}, \quad e^{-}=H^{-3 / 8}\left(d x^{-}+\frac{1}{2} K d x^{+}\right), \quad e^{i}=H^{1 / 8} d z^{i}
$$

where the local Lorentz metric in the tangent frame is taken to be

$$
\eta_{+-}=\eta_{-+}=1, \quad \eta_{i j}=\delta_{i j}
$$

We find that the torsion-free spin connection is then given by

$$
\begin{aligned}
& \omega^{+}{ }_{i}=-\frac{3}{8} H^{-9 / 8} H_{i} e^{+}, \quad \omega^{-}{ }_{i}=-\frac{3}{8} H^{-9 / 8} H_{i} e^{-}+\frac{1}{2} H^{-1 / 8} K_{i} e^{+}, \\
& \omega^{i}{ }_{j}=-\frac{1}{8} H^{-9 / 8}\left(H_{i} e^{j}-H_{j} e^{i}\right)
\end{aligned}
$$

where $H_{i} \equiv \partial_{i} H$ and $K_{i} \equiv \partial_{i} K$. It follows from (104) that the Lorentz-covariant exterior derivative is $\nabla=d+\frac{1}{4} \omega^{A B} \Gamma_{A B}$, from which one can read off the vielbein components $\nabla_{A}$ via $\nabla=e^{A} \nabla_{A}$, is given by

$$
\nabla=d-\frac{3}{16} H^{-9 / 8} H_{i} \Gamma_{+i} e^{+}-\frac{3}{16} H^{-9 / 8} H_{i} \Gamma_{-i} e^{-}+\frac{1}{4} H^{-1 / 8} K_{i} \Gamma_{-i} e^{+}-\frac{1}{16} H^{-9 / 8} H_{i} \Gamma_{i j} e^{j}
$$

From (104), we find that the frame components of the Ricci tensor are given by

$$
\begin{aligned}
& R_{+-}=+\frac{3}{8} H^{-5 / 4} \square H-\frac{3}{8} H^{-9 / 4} H_{i} H_{i}, \quad R_{++}=-\frac{1}{2} H^{-1 / 4} \square K, \quad R_{--}=0, \\
& R_{i j}=-\frac{1}{8} H^{-5 / 4} \square H \delta_{i j}+\frac{1}{8} H^{-9 / 4} H_{k} H_{k} \delta_{i j}-\frac{3}{8} H^{-9 / 4} H_{i} H_{j},
\end{aligned}
$$

where $\square \equiv \partial_{i} \partial_{i}$ is the Laplacian in the flat eight-dimensional transverse space.

\subsection{The type IIB supergravity solution}

We seek here a solution describing the intersection of a string and a pp-wave in the type IIB theory. There is a standard well-known such solution for source-free strings and waves, and so we can take this as our starting point, and look for a generalisation in which the 5 -form is taken to have a constant background value, as in the pp-wave. Thus we consider a configuration where the metric is given by (100), and the field strengths are

$$
F_{(5)}=\mu d x^{+} \wedge \Phi_{(4)}, \quad G_{(3)}=d H^{-1} \wedge d x^{+} \wedge d x^{-}, \quad \phi=-\frac{1}{2} \log H
$$


where $G_{(3)}$ is the NS-NS 3-form, and $\Phi_{(4)}$ is a constant self-dual tensor in the transverse space $\mathbb{R}^{8}$, whose coordinates are the $z^{i}$. The novel feature, for an intersection, is the inclusion of the 5-form term.

The type IIB equations of motion for the fields that we are taking to be non-zero are

$$
\begin{aligned}
& d\left(e^{-\phi} * G_{(3)}\right)=0, \quad * F_{(5)}=F_{(5)}, \quad d F_{(5)}=0, \\
& R_{A B}=\frac{1}{2} \partial_{A} \phi \partial_{B} \phi+\frac{1}{4} e^{-\phi}\left(G_{A C D} G_{B} C D-\frac{1}{12} G_{C D E} G^{C D E} g_{A B}\right)+\frac{1}{96} F_{A C D E F} F_{B} C D E F, \\
& \square \phi=-\frac{1}{12} e^{-\phi} G_{A B C} G^{A B C} .
\end{aligned}
$$

After some straightforward algebra, we find that these are satisfied provided that the functions $H$ and $K$ satisfy

$$
\square H=0, \quad \square K=-\frac{1}{48} \mu^{2}\left|\Phi_{(4)}\right|^{2} .
$$

If we set the constant $\mu=0$, we just get back the standard solution for the intersection of a string and a wave. The new feature here is that we still obtain a solution if we take $\mu$ to be non-zero. Thus, in particular, we have a solution with

$$
K=c_{0}-\sum_{i} \mu_{i}^{2} z_{i}^{2}, \quad H=1+\frac{Q}{r^{6}},
$$

where $\sum_{i} \mu_{i}^{2}=\frac{1}{96} \mu^{2}\left|\Phi_{(4)}\right|^{2}$, and $r^{2}=z_{i}^{2}$. (We could, of course, choose more complicated solutions for $H$ and for $K$.)

\subsection{Supersymmetry of the type IIB string on a pp-wave}

The supersymmetry transformation rules for the type IIB gravitini $\psi_{M}$ and dilatini $\lambda$, in the background involving the dilaton, NS-NS 3-form and the 5-form are

$$
\begin{aligned}
\delta \psi_{M} & =\nabla_{M} \epsilon+\frac{\mathrm{i}}{192} F_{M N_{1} \cdots N_{4}} \Gamma^{N_{1} \cdots N_{4}} \epsilon-\frac{1}{96} e^{-\frac{1}{2} \phi} G_{N P Q}\left(\Gamma_{M}^{N P Q}-9 \delta_{M}^{N} \Gamma^{P Q}\right) \epsilon^{*} \\
\delta \lambda & =\Gamma^{M} \partial_{M} \phi \epsilon^{*}-\frac{1}{12} e^{-\frac{1}{2} \phi} G_{M N P} \Gamma^{M N P} \epsilon .
\end{aligned}
$$

It is helpful to define

$$
U \equiv \frac{1}{5 !} F_{N_{1} \cdots N_{5}} \Gamma^{N_{1} \cdots N_{5}}, \quad V \equiv \frac{1}{3 !} G_{M N P} \Gamma^{M N P}
$$

in terms of which the gravitino transformation rule becomes

$$
\delta \psi_{M}=\nabla_{M} \epsilon+\frac{\mathrm{i}}{16}\left\{\Gamma_{M}, U\right\} \epsilon-\frac{1}{16} e^{-\frac{1}{2} \phi}\left(\Gamma_{M} V+2 V \Gamma_{M}\right) \epsilon^{*}=0 .
$$

In our background, where $F_{(5)}$ is given in (107), we have

$$
U=\mu \Gamma_{-} W, \quad W \equiv \frac{1}{4 !} \Phi_{i j k \ell} \Gamma^{i j k \ell}
$$


From the dilatino transformation rule in (111) we obtain

$$
\delta \lambda=-\frac{1}{2} H^{-9 / 8} H_{i} \Gamma_{i}\left(\epsilon^{*}+\Gamma_{+-} \epsilon\right)=0,
$$

implying that the string imposes the projection condition

$$
\epsilon^{*}+\Gamma_{+-} \epsilon=0
$$

Using this, we then find that the transverse components $\delta \psi_{i}=0$ of the gravitino transformation rules imply

$$
\epsilon=H^{-\frac{3}{16}} \epsilon_{0}, \quad \partial_{i} \epsilon_{0}-\frac{\mathrm{i}}{16} \mu \Gamma_{-}\left[\Gamma_{i}, W\right] \epsilon_{0}=0 .
$$

From $\delta \psi_{+}=0$ we get

$$
\Gamma_{+}\left(\epsilon-\epsilon^{*}\right)=0, \quad H^{1 / 2} \partial_{+} \epsilon+\frac{1}{4} K_{i} \Gamma_{-} \Gamma_{i} \epsilon+\frac{\mathrm{i}}{8} \mu W \epsilon=0,
$$

and finally from $\delta \psi_{-}=0$ we obtain

$$
\Gamma_{-}\left(\epsilon+\epsilon^{*}\right)=0, \quad \partial_{-} \epsilon=0 .
$$

These conditions can be summarised as follows. We have the usual $\epsilon=H^{-3 / 16} \epsilon_{0}$ factor and the projection conditions

$$
\Gamma_{+}\left(\epsilon-\epsilon^{*}\right)=0, \quad \Gamma_{-}\left(\epsilon+\epsilon^{*}\right)=0
$$

for a string in type IIB theory. The remaining equations

$$
\partial_{i} \epsilon_{0}-\frac{\mathrm{i}}{16} \mu \Gamma_{-}\left[\Gamma_{i}, W\right] \epsilon_{0}=0, \quad H^{1 / 2} \partial_{+} \epsilon_{0}+\frac{1}{4} K_{i} \Gamma_{-} \Gamma_{i} \epsilon_{0}+\frac{\mathrm{i}}{8} \mu W \epsilon_{0}=0, \quad \partial_{-} \epsilon_{0}=0
$$

are like those for a pp-wave, except for the factor $H^{1 / 2}$ that multiplies $\partial_{+}$. This means that we can only have Killing spinors that are independent of $x^{+}$.

Analysis of the content of equations (121) is analogous to the discussion for pure ppwaves given in [7]. The first equation implies that $\epsilon_{0}$ can be written as

$$
\epsilon_{0}=\left(1+\frac{\mathrm{i}}{16} \mu\left[\Gamma_{i}, W\right] \Gamma_{-}\right) \chi
$$

where $\chi$ is independent of $z_{i}$. In the present context, with a superimposed string, $\chi$ is therefore a constant spinor. It then follows from (121) that $\chi$ must satisfy

$$
W \chi=0, \quad\left(\mu^{2} z_{i} W^{2}+32 K_{i}\right) \Gamma_{i} \Gamma_{-} \chi=0 .
$$




\subsection{The type IIA supergravity solution}

We shall look for an intersecting solution analogous to the solution (107) that we found in the type IIB theory. We again take the metric to be (100), and for the field strengths we take

$$
F_{(4)}=\mu d x^{+} \wedge \Phi_{(3)}, \quad F_{(3)}=d H^{-1} \wedge d x^{+} \wedge d x^{-}, \quad \phi=-\frac{1}{2} \log H
$$

where we have the novel feature of the $F_{(4)}$ term given in terms of the constant 3-form $\Phi_{3}$ on the flat $\mathbb{R}^{8}$ transverse space. Using the results for the Ricci tensor presented in (106), we find after straightforward algebra that the type IIA equations of motion are satisfied if $H$ and $K$ satisfy

$$
\square H=0, \quad \square K=-\frac{1}{6} \mu^{2}\left|\Phi_{3}\right|^{2},
$$

where $\square=\partial_{i} \partial_{i}$ is the Laplacian in the flat transverse space. As in the type IIB example, we can take

$$
K=c_{0}-\sum_{i} \mu_{i}^{2} z_{i}^{2}, \quad H=1+\frac{Q}{r^{6}},
$$

where $\sum_{i} \mu_{i}^{2}=\frac{1}{12} \mu^{2}\left|\Phi_{(3)}\right|^{2}$, and $r^{2}=z_{i}^{2}$.

Note that we can lift this intersecting solution back to $D=11$ supergravity, by using the standard Kaluza-Klein reduction

$$
\begin{aligned}
d \hat{s}_{11}^{2} & =e^{-\frac{1}{6} \phi} d s_{10}^{2}+e^{\frac{4}{3} \phi}\left(d z_{9}+\mathcal{A}_{1}\right)^{2}, \\
\hat{A}_{(3)} & =A_{(3)}+\left(d y+\mathcal{A}_{(1)}\right) \wedge A_{(2)} .
\end{aligned}
$$

Since we have $\mathcal{A}_{(1)}=0$ here, we find that the lifting just gives

$$
\begin{aligned}
d \hat{s}_{11}^{2} & =H^{-2 / 3}\left(2 d x^{+} d x^{-}+K d x^{+^{2}}+d z_{10}^{2}\right)+H^{1 / 3} d z_{i}^{2}, \\
\hat{F}_{(4)} & =\mu d x^{+} \wedge \Phi_{(3)}+d y \wedge d H^{-1} \wedge d x^{+} \wedge d x^{-} .
\end{aligned}
$$

(Note that $z_{i}$ has $1 \leq i \leq 8$, denoting the transverse coordinates in the 8-dimensional transverse space, while $z_{10}$ denotes the extra coordinate of eleven dimensions.) This describes a membrane living in a pp-wave background in eleven dimensions.

\subsection{Supersymmetry of the type IIA string on a pp-wave}

This can be studied most simply by lifting the solution to eleven-dimensional supergravity. In $D=11$, we choose the natural vielbein basis for the metric in (128), giving

$$
\hat{e}^{10}=H^{-1 / 3} d z_{10},
$$




$$
\begin{aligned}
\hat{e}^{i} & =H^{1 / 24} e^{i}=H^{1 / 6} d z^{i}, \quad 1 \leq i \leq 8, \\
\hat{e}^{+} & =H^{1 / 24} e^{+}=H^{-1 / 3} d x^{+}, \\
\hat{e}^{-} & =H^{1 / 24} e^{-}=H^{-1 / 3}\left(d x^{+}+\frac{1}{2} K d x^{-}\right) .
\end{aligned}
$$

Using standard Kaluza-Klein formulae, we can express the spin connection $\hat{\omega}^{A B}$ in $D=11$ in terms of $D=10$ quantities, and hence we find that $\hat{\nabla} \equiv d+\frac{1}{4} \hat{\omega}^{A B} \Gamma_{A B}$ is given by

$$
\hat{\nabla}=d-\frac{1}{12} H^{-7 / 6} H_{i}\left(2 \Gamma_{+i} \hat{e}^{+}+2 \Gamma_{-i} \hat{e}^{-}+\Gamma_{i j} \hat{e}^{j}\right)+\frac{1}{4} H^{-1 / 6} K_{i} \Gamma_{-i} \hat{e}^{+}+H^{-7 / 6} \Gamma_{i} \Gamma_{\underline{10}} \hat{e}^{10},
$$

where we are denoting by $\Gamma_{\underline{10}}$ the eleven-dimensional Dirac matrix associated with the extra coordinate $z_{10}$ of the M-theory circle. (We underline the "10" to avoid the danger of confusion between $\Gamma_{10}$ and $\Gamma_{1} \Gamma_{0}$.) We reserve the subscript "9" on a Dirac matrix for the spatial direction associated with the $x^{ \pm} \equiv\left(x^{9} \pm x^{0}\right) / \sqrt{2}$ coordinates.

The gravitino transformation rule is given by $\delta \hat{\psi}_{A}=\hat{\nabla}_{A} \epsilon+\hat{T}_{A} \epsilon$, where

$$
\hat{T}_{A}=-\frac{1}{288} \Gamma_{A}{ }^{B C D E} \hat{F}_{B C D E}+\frac{1}{36} \hat{F}_{A B C D} \Gamma^{B C D}
$$

The $D=11$ frame components of the field strength $\hat{F}_{(4)}$ can be read off from (128) and (129), giving

$$
\hat{F}_{+i j k}=\mu H^{-1 / 6} \Phi_{i j k}, \quad \hat{F}_{i \underline{10}+-}=H^{-7 / 6} H_{i} .
$$

(Again, we underline the "10" subscript here to avoid potential confusion with subscripts "1" followed by "0."

Substituting into the $D=11$ supersymmetry transformation rule, we find that $\delta \hat{\psi}_{A}=0$ implies

$$
\begin{aligned}
& \partial_{10} \epsilon+\frac{\mathrm{i}}{12} \mu H^{-1 / 2} W \Gamma_{\underline{10},-} \epsilon+\frac{1}{6} H_{i} \Gamma_{i}\left(\Gamma_{\underline{10}}-\Gamma_{-+}\right) \epsilon=0 \\
& \partial_{i} \epsilon+\frac{\mathrm{i}}{24} \mu\left(\Gamma_{i} W+3 W \Gamma_{i}\right) \Gamma_{-} \epsilon+\frac{1}{12} H^{-1}\left[2 H_{i} \Gamma_{\underline{10}} \Gamma_{-+}+H_{j} \Gamma_{i j}\left(1-\Gamma_{\underline{10}} \Gamma_{-+}\right)\right] \epsilon=0, \\
& \partial_{-} \epsilon+\frac{1}{6} H^{-3 / 2} H_{i} \Gamma_{i} \Gamma_{-}\left(1+\Gamma_{\underline{10}}\right) \epsilon=0, \\
& \partial_{+} \epsilon-\frac{1}{2} K \partial_{-} \epsilon \\
& +\frac{1}{4} H^{-1 / 2}\left[K_{i} \Gamma_{-i}-\frac{1}{3} \mu W\left(1+\Gamma_{-} \Gamma_{+}\right)\right] \epsilon+\frac{1}{6} H^{-3 / 2} H_{i} \Gamma_{i} \Gamma_{+}\left(1-\Gamma_{\underline{10}}\right) \epsilon=0,
\end{aligned}
$$

where we have defined

$$
W \equiv \frac{i}{6} \Phi_{i j k} \Gamma_{i j k}
$$

The four lines come from $\delta \hat{\psi}_{10}=0, \delta \hat{\psi}_{i}=0, \delta \hat{\psi}_{-}=0, \delta \hat{\psi}_{+}=0$ respectively. Note that indices on $\partial$ are all coordinate indices, while those on the Dirac matrices are all tangentframe indices. 
It is easy to see from the equations above that we must have

$$
\begin{aligned}
& \Gamma_{-}\left(1+\Gamma_{\underline{10}}\right) \epsilon=0, \quad \Gamma_{+}\left(1-\Gamma_{\underline{10}}\right) \epsilon=0, \\
& \partial_{10} \epsilon=0, \quad \partial_{-} \epsilon=0, \quad \partial_{+} \epsilon=0,
\end{aligned}
$$

and that if we define $\epsilon=H^{-1 / 6} \eta$, then $\eta\left(z_{i}\right)$ must satisfy

$$
\partial_{i} \eta+\frac{\mathrm{i}}{24} \mu\left(\Gamma_{i} W+3 W \Gamma_{i}\right) \Gamma_{-} \eta=0, \quad K_{i} \Gamma_{-i} \eta-\frac{\mathrm{i}}{3} \mu W\left(1+\Gamma_{-} \Gamma_{+}\right) \eta=0 .
$$

The first line in (135) represents the usual projections that one encounters for a string solution, implying a half-breaking of supersymmetry. The conditions in (136) are the same as those encountered in [8] for pure pp-waves, except that here we have the added requirement of $x^{+}$independence. As described in [8], the first equation in (136) implies that

$$
\eta=\left(1-\frac{\mathrm{i}}{24} \mu\left(\Gamma_{i} W+3 W \Gamma_{i}\right) \Gamma_{-}\right) \chi
$$

where $\chi$ is independent of the $z_{i}$. Since here we also have $\partial_{+} \epsilon=0$, it follows, using arguments analogous to those in [8], that $\chi$ must satisfy

$$
W \chi=0, \quad\left(\mu^{2} z_{i} W^{2}+8 K_{i}\right) \Gamma_{i} \chi=0 .
$$

Following [11], we shall define the chirality operator $\Gamma_{11} \equiv-\Gamma_{\underline{10}}$, where it will be recalled that $\Gamma_{\underline{10}}$ denotes the eleven-dimensional Dirac matrix in the direction of the M-theory circle. Multiplying the expressions on the first line in (135) by $\Gamma_{9}$, they can be written as

$$
\left(1+\Gamma_{09}\right)\left(1-\Gamma_{09} \Gamma_{*}\right) \epsilon=0, \quad\left(1-\Gamma_{09}\right)\left(1+\Gamma_{09} \Gamma_{*}\right) \epsilon=0,
$$

where $\Gamma_{*} \equiv \Gamma_{1} \cdots \Gamma_{8}$ as in (61). Adding and subtracting these equations, we find that the conditions on the first line in (135) imply and are implied by

$$
\left(1-\Gamma_{*}\right) \epsilon=0 .
$$

Thus we see that the string component of the string/pp-wave intersection in the type IIA theory imposes precisely the gamma-matrix projection condition that we used in the physical-gauge fixing in section 5.1.

\subsection{Strings on pp-waves with odd numbers of Killing spinors}

Here, we study the number of Killing spinors that can occur in the type IIA and type IIB strings in pp-wave backgrounds. As we stated earlier, the type IIA and type IIB cases are 
related by T-duality. Thus it suffices to study the type IIA example. We take the constant 3 -form to be $[7,8]$

$$
\begin{aligned}
\phi_{(3)}= & m_{1} d z^{123}+m_{2} d z^{145}+m_{3} d z^{167}+m_{4} d z^{246} \\
& +m_{5} d z^{257}+m_{6} d z^{347}+m_{7} d z^{356}
\end{aligned}
$$

As we saw in (6.4), for both the standard and supernumerary Killing spinors we must have $W \chi=0$, which implies that

$$
m_{1}+m_{2}+m_{3}-m_{4}+m_{5}+m_{6}+m_{7}=0
$$

The standard Killing spinors satisfy the three conditions

$$
\Gamma_{-} \chi=0, \quad W \chi=0, \quad\left(1-\Gamma_{\underline{10}}\right) \chi=0 .
$$

Since $\Gamma_{-}, \Gamma_{\underline{10}}$ and $W$ commute, it follows that the total number of Killing spinors is a quarter of the number of spinors annihilated by $W$.

In the case of pure pp-waves with no string present, the number of standard Killing spinors is 16, and is always independent of the structure of $W$. The detailed structure of $W$ determines their $x^{+}$dependence, with only those that are annihilated by $W$ being independent of $x^{+}$. In the present case, where there is a string on the pp-wave background, we saw in section 6.4 that the Killing spinors must be $x^{+}$-independent, and so it follows that even the number of standard Killing spinors will now depend on the structure of $W$. It is straightforward to see that a generic set of $m_{i}$ satisfying the condition (142) will give rise to one standard Killing spinor, with a maximum of four standard Killing spinors being achievable for suitable special choices of the $m_{i}$.

The conditions for supernumerary Killing spinors are slightly more restrictive; they are given by

$$
\Gamma_{+} \chi=0, \quad W \chi=0, \quad\left(1+\Gamma_{\underline{10}}\right) \chi=0, \quad\left(\mu^{2} z_{i} W^{2}+8 K_{i}\right) \Gamma_{i} \chi=0 .
$$

For a generic set of $m_{i}$ satisfying (142), there is one supernumerary Killing spinor, with a maximum of four for special values of $m_{i}$.

Let us look at some examples in detail. If there is only one non-vanishing $m_{i}, W$ has no zero eigenvalues, and so there are neither standard nor supernumerary Killing spinors.

For two non-vanishing $m_{i}$, say $m_{1}$ and $m_{2}$, we can obtain Killing spinors if we choose $m_{1}+m_{2}=0$. There are then four standard Killing spinors and four supernumerary Killing spinors. 
For three non-vanishing $m_{i}$, say $m_{1}, m_{2}$ and $m_{3}$, there exist Killing spinors if $m_{1}+$ $m_{2}+m_{3}=0$. For generic such choices, there are two standard Killing spinors and two supernumerary Killing spinors.

In general, when there are four or more non-vanishing $m_{i}$, there will be one standard Killing spinor and one supernumerary Killing spinor provided that the $m_{i}$ satisfy (142). However, more Killing spinors can arise in special cases. For example, if we have $m_{1}=1$, $m_{2}=-1, m_{3}=1$ and $m_{4}=1$, we have three standard Killing spinors and one supernumerary Killing spinor. For a more complicated example, $m_{1}=1, m_{2}=-1, m_{3}=1, m_{4}=1$, $m_{5}=1, m_{6}=-1$, we have four standard Killing spinors and one supernumerary Killing spinor, giving a total of five Killing spinors. If $m_{1}=2, m_{2}=-2, m_{3}=1, m_{4}=1, m_{5}=1$, $m_{6}=-1$, we have two standard Killing spinors and one supernumerary Killing spinor, giving a total of three. These latter two examples are of interest since the total number of Killing spinors is odd.

\subsection{Further solutions for strings in pp-waves}

\section{A type IIB generalisation}

For this example, we again take the metric and dilaton to be

$$
\begin{aligned}
d s_{10}^{2} & =H^{-3 / 4}\left(2 d x^{+} d x^{-}+K d x^{+^{2}}\right)+H^{1 / 4} d z_{i}^{2} \\
\phi & =-\frac{1}{2} \log H
\end{aligned}
$$

but now the solution is supported by the NS-NS and R-R 3-form field strengths $G_{(3)}$ and $F_{(3)}$ :

$$
G_{(3)}=d H^{-1} \wedge d x^{+} \wedge d x^{-}, \quad F_{(3)}=\mu d x^{+} \wedge \Phi_{(2)},
$$

where $\Phi_{(2)}$ is a constant 2-form in the eight-dimensional transverse space. It can be verified that this solves the type IIB equations of motion provided that $H$ and $K$ satisfy

$$
\square H=0, \quad \square K=-\frac{1}{2} \mu^{2}\left|\Phi_{(2)}\right|^{2}
$$

in the transverse space.

\section{A type IIA generalisation}

We can generalise the type IIA string/pp-wave given in (124) by adding in a constant R-R 2-form field strength term, so that we now have

$$
\begin{aligned}
& d s_{10}^{2}=H^{-3 / 4}\left(2 d x^{+} d x^{-}+K d x^{+^{2}}\right)+H^{1 / 4} d z_{i}^{2}, \quad \phi=-\frac{1}{2} \log H \\
& F_{(4)}=\mu d x^{+} \wedge \Phi_{(3)}, \quad F_{(3)}=d H^{-1} \wedge d x^{+} \wedge d x^{-}, \quad F_{2}=\tilde{\mu} d x^{+} \wedge \Phi_{(1)} .
\end{aligned}
$$


Substituting into the type IIA equations of motion, we find that they are satisfied provided that $H$ and $K$ satisfy

$$
\square H=0, \quad \square K=-\frac{1}{6} \mu^{2}\left|\Phi_{(3)}\right|^{2}-\tilde{\mu}^{2}\left|\Phi_{(1)}\right|^{2} .
$$

Some similar solutions, corresponding to cases of D-branes in pp-wave backgrounds that originate from $\mathrm{AdS}_{3} \times S^{3} \times R^{4}$, can be found in $[15,16]$.

\section{Conclusions}

In this paper, we have studied the relation between the occurrence of Killing spinors in a pp-wave background, and the worldsheet supersymmetry of the associated string action after gauge fixing. In the light-cone gauge, the string action gives a free theory with masses for the bosonic and fermionic fields [6].

The Killing spinors in the pp-wave background comprise 16 "standard" Killing spinors, which are present in arbitrary pp-wave backgrounds, plus an additional $N_{\text {sup }}$ supernumerary Killing spinors, with $0 \leq N_{\text {sup }} \leq 16$, which arise only in special cases. We showed that in the light-cone gauge, none of the 16 standard Killing spinors give rise to any linearly-realised worldsheet supersymmetries. This is because the projection condition for the linearly-realised worldsheet supersymmetries arising from the local kappa symmetry and target-spacetime supersymmetry after light-cone gauge fixing is orthogonal to the projection condition satisfied by the standard Killing spinors. By contrast, the supernumerary Killing spinors satisfy the opposite projection condition, and so these are precisely the ones that are associated with linearly-realised worldsheet supersymmetries in the light-cone gauge.

Depending upon the specific details of a pp-wave solution that admits supernumerary Killing spinors, these Killing spinors may either depend upon the coordinate $x^{+}$, or else be independent of $x^{+}$. Since $x^{+}$is set equal to $\tau$ in the light-cone gauge, the question of $x^{+}$dependence determines whether or not the associated linearly-realised worldsheet supersymmetries commute with the Hamiltonian. If they do commute (i.e. when the Killing spinors do not depend upon $x^{+}$), then the linear supersymmetry ensures that the bosonic and fermionic coordinates will have paired sets of equal mass terms. If the supernumerary Killing spinors instead depend upon $x^{+}$, then even though there are still linearly-realised worldsheet supersymmetries, they no longer imply any equality of the boson and fermion masses.

Since the light-cone gauge provides the standard passage between the Green-Schwarz and the Neveu-Schwarz-Ramond formalisms, the considerations of this paper could lead to 
a better understanding of the NSR formalism in the presence of Ramond-Ramond backgrounds, such as the constant RR forms that we have discussed. The passage to the NSR formalism endows a specific worldsheet $(1,1)$ supersymmetry with a special importance, being the rigid gauge-fixed remnant of the NSR $(1,1)$ local worldsheet supersymmetry. This passage makes use of Killing spinors on the spacetime background for the light-cone gauge fixed theory in order to change the fermionic worldsheet variables from spinor to vector representation of the transverse-space structure group (c.f., e.g. [17]). ${ }^{5}$

Additional NSR worldsheet supersymmetries beyond the original $(1,1)$ arise depending on the geometry of the background. For example, if the transverse geometry is Kähler, one obtains $(2,2)$ supersymmetry; if it is hyper-Kähler, one obtains $(4,4)$ supersymmetry, etc. These additional NSR supersymmetries are distinguished from the initial $(1,1)$ supersymmetry in that they all involve complex structures on the target manifold of the resulting NSR worldsheet sigma model. Thus, the linearly realised and $x^{+}$independent lightcone gauge supersymmetries that we have discussed in this paper can be expected to translate to rigid NSR supersymmetries for superstrings propagating on the class of pp-wave backgrounds that we have discussed.

In this paper, we have found that a quite different situation arises if one chooses the physical gauge instead of the light-cone gauge. We showed that in this case the projection conditions for the residual worldsheet supersymmetries that arise from the original kappa symmetry and target-spacetime supersymmetry now imply that the linearly-realised worldsheet supersymmetries come both from standard and from supernumerary Killing spinors. We also showed that, consistently with this, there are in fact no mass terms at all in the string worldsheet action in the physical gauge, either for the bosonic or the fermionic coordinates. This emphasises the fact that the mass terms in the string worldsheet actions in the light-cone gauge can be viewed as artefacts of the specific gauge-fixing procedure.

We also obtained new supergravity solutions that describe strings in pp-wave backgrounds. These can be viewed as the classical solitonic realisations of string actions whose target spacetimes are pp-waves. The supergravity solutions naturally describe these string actions in the physical gauge, since the $x^{ \pm}$coordinates of the pp-wave are the same as the worldsheet coordinates of the string component of the classical solution. We find that indeed the supersymmetries of these classical supergravity solutions coincide with the super-

\footnotetext{
${ }^{5} \mathrm{An}$ alternative approach is to employ the bosonisation procedure for the worldsheet fermions. Bosonisation provides a direct map between the worldsheet fields in the NSR formalism, where they transform as space-time vectors, and the worldsheet fields in the GS formalism, where they transform as space-time spinors, c.f., e.g. [18].
} 
symmetries that one finds for the string actions in the corresponding pp-wave background, upon imposition of the physical gauge conditions. In particular, we find that there can be odd numbers of Killing spinors in these solutions.

\section{Acknowledgments}

K.S.S. would like to thank Chris Hull for discussions. M.C., C.N.P. and K.S.S. thank the Isaac Newton Institute and CERN, C.N.P. and K.S.S. thank the Ecole Normale, Paris, M.C. thanks the New Center for Theoretical Physics at Rutgers University and K.S.S. thanks the Institut des Hautes Etudes Scientifiques, for hospitality and support at various times during the course of this work.

\section{References}

[1] J. Kowalski-Glikman, Vacuum States In Supersymmetric Kaluza-Klein Theory, Phys. Lett. B134, 194 (1984).

[2] M. Blau, J. Figueroa-O'Farrill, C. Hull and G. Papadopoulos, A new maximally supersymmetric background of IIB superstring theory, JHEP 0201 (2002) 047, hepth/0110242.

[3] M. Blau, J. Figueroa-O'Farrill, C. Hull and G. Papadopoulos, Penrose limits and maximal supersymmetry, hep-th/0201081.

[4] R. Penrose, Any space-time has a plane wave as a limit, in Differential geometry and relativity, Reidel, Dordrecht, 1976.

[5] R.R. Metsaev, Type IIB Green-Schwarz superstring in plane wave Ramond-Ramond background, Nucl. Phys. B625, 70 (2002), hep-th/0112044.

[6] D. Berenstein, J. Maldacena and H. Nastase, Strings in flat space and pp waves from $N=4$ super Yang Mills, hep-th/0202021.

[7] M. Cvetič, H. Lü and C.N. Pope, Penrose limits, pp-waves and deformed M2-branes, hep-th/0203082.

[8] M. Cvetič, H. Lü and C.N. Pope, M-theory pp-waves, Penrose limits and supernumerary supersymmetries, hep-th/0203229, to appear in Nucl. Phys. B. 
[9] J. Gauntlett and C.M. Hull, pp-waves in 11-dimensions with extra supersymmetry, JHEP 0206, 013 (2002), hep-th/0203255.

[10] H. Lü and J.F. Vazquez-Poritz, Penrose limits of non-standard brane intersections, Class. Quant. Grav. 19, 4059 (2002), hep-th/0204001.

[11] M. Cvetič, H. Lü, C.N. Pope and K.S. Stelle, T-duality in the Green-Schwarz formalism, and the massless/massive IIA duality map, Nucl. Phys. B573, 149 (2000), hep-th/9907202.

[12] B. de Wit, K. Peeters and J. Plefka, Superspace geometry for supermembrane backgrounds, Nucl. Phys. B532 (1998) 99, hep-th/9803209.

[13] M.J. Duff, P.S. Howe, T. Inami and K.S. Stelle, Superstrings in D $=10$ from supermembranes in $D=11$, Phys. Lett. 191B (1987) 70.

[14] R. Corrado, N. Halmagyi, K.D. Kennaway and N.P. Warner, Penrose limits of RG fixed points and pp-waves with background fluxes, hep-th/0205314.

[15] A. Kumar, R.R. Nayak and Sanjay, D-brane solutions in pp-wave background, Phys. Lett. B541, 183 (2002), hep-th/0204025.

[16] A. Biswas, A. Kumar and K.L. Panigrahi, $p-p^{\prime}$ branes in pp-wave background, hepth/0208042.

[17] C.M. Hull, Compactifications of the heterotic superstring, Phys. Lett. B178, 357 (1986);

M.D. Freeman, C.N. Pope, C.M. Hull and K.S. Stelle, Space-time versus world sheet supersymmetry in the heterotic string, Phys. Lett. B185, 351 (1987).

[18] M.E. Peskin, Introduction To String And Superstring Theory. 2, SLAC-PUB-4251, Lectures presented at the 1986 Theoretical Advanced Study Institute in Particle Physics, Santa Cruz, Calif., Jun 23 - Jul 19, 1986. 